\title{
Contour-based object identification and segmentation: Stimuli, norms and data, and software tools
}

\author{
JOERI DE WINTER and JOHAN WAGEMANS \\ University of Leuven, Leuven, Belgium
}

\begin{abstract}
We summarize five studies of our large-scale research program, in which we examined aspects of contour-based object identification and segmentation, and we report on the stimuli we used, the norms and data we collected, and the software tools we developed. The stimuli were outlines derived from the standard set of line drawings of everyday objects by Snodgrass and Vanderwart (1980). We used contour curvature as a major variable in all the studies. The total number of 1,500 participants produced very solid, normative identification rates of silhouettes and contours, straight-line versions, and fragmented versions, and quite reliable benchmark data about saliency of points and object segmentation into parts. We also developed several software tools to generate stimuli and to analyze the data in nonstandard ways. Our stimuli, norms and data, and software tools have great potential for further exploration of factors influencing contour-based object identification, and are also useful for researchers in many different disciplines (including computer vision) on a wide variety of research topics (e.g., priming, agnosia, perceptual organization, and picture naming). The full set of norms, data, and stimuli may be downloaded from www.psychonomic.org/archive/.
\end{abstract}

Identifying objects in line drawings is remarkably easy for humans. If the object is drawn from a well chosen viewpoint, not much information appears to be lost in the projection of its 3-D shape onto a single 2-D image or in the further reduction of the continuous distribution of spectral or gray-level values to a limited number of discrete black lines. Moreover, in many cases the outline, without the interior features, seems to contain sufficient information to derive the object's shape and identity. Although this fascinating problem has been studied for a long time (see, e.g., Attneave, 1954), research gained renewed momentum from new theoretical proposals in the 1980 s (see, e.g., Biederman, 1987; Hoffman \& Richards, 1984; Lowe, 1987; Richards \& Hoffman, 1985; Ullman, 1989).

Many of these theories attribute a special role to contour curvature. More specifically, Attneave (1954) argued that information about object shape is not distributed homogeneously along the contour but is concentrated at points where contour curvature reaches its extreme values (i.e., curvature extrema) either positively (i.e., maxima, or

This research program is carried out with financial support of Grant OT/00/007 of the University of Leuven and Grant G.0189.02 of the Fund for Scientific Research, Flanders. A number of collaborators have contributed to parts of the studies reported here. We thank all of them for their contributions: Tom Beckers, Wim Notebaert, Hans Op de Beeck, Sven Panis, Annemie Ploeger, Toon Sykora, Eva Van de gaer, and Peter Vanroose. The manuscript was improved thanks to very useful comments from two anonymous reviewers and the editor, Jonathan Vaughan. Correspondence concerning this article should be addressed to J. Wagemans, University of Leuven, Department of Psychology, Tiensestraat 102, Leuven B-3000, Belgium (e-mail: johan.wagemans@psy.kuleuven.ac.be).
$M+)$ or negatively (i.e., minima, or $m-$ ). Hoffman and Richards (1984) and Biederman (1987) argued that objects are recognized on the basis of their parts and have proposed that objects are segmented into parts at regions of deep concavities or minima of curvature (i.e., the minima rule). Richards and Hoffman (1985) proposed a codon theory in which shapes are encoded as sequences of primitive contour fragments, or codons, each of which is determined by its curvature singularities (i.e., $M+, m-$, and inflections $[I]$, where curvature passes through zero in the transition between convex and concave regions).

These theories are interesting because they are quite specific about the nature of the information that is important for shape and object encoding, segmentation, and identification. These curvature singularities can be defined and located with mathematical precision. However, the empirical support has lagged behind theoretical ideas because the ideas have usually been tested on a limited number of shapes and objects, using small groups of observers. For example, the minima rule of segmentation has been tested on a small number of random shapes (eight by Braunstein, Hoffman, \& Saidpour, 1989) or contour drawings of everyday objects (seven by Siddiqi, Tresness, \& Kimia, 1996), using only 8-17 and 5-14 participants, respectively. Attneave's (1954) demonstration on the role of curvature extrema for shape encoding (based on an unpublished study using 16 random shapes and 80 participants) was replicated almost half a century later using 12 silhouettes of sweet potatoes and 12 participants (Norman, Phillips, \& Ross, 2001). Attneave's demonstration that a sleeping cat could still be recognized when the curved lines were replaced by straight lines connect- 
ing visually salient curvature extrema awaits serious experimental confirmation on other everyday objects.

For these reasons, we have embarked upon a largescale research program in which we have tested several ideas about the role of curvature singularities for object identification and segmentation, using much larger sets of stimuli and groups of participants. Along the road, this research program has produced stimuli as well as norms and data that other researchers may want to use for their studies. ${ }^{1}$ This special issue offers an excellent opportunity to make them available to the research community (for an overview, see Tables 1 and 2, respectively). We will use our research questions as a guideline in this overview, but we concentrate on the electronic archiving aspects and refer the reader to the separate research reports for further details concerning theoretical and empirical aspects.

\section{Outline}

Before we describe the separate studies, it is useful to start with a brief overview providing some background of the whole research program and some guidance in understanding the rationale behind it. The theoretical point of departure, as has already been indicated, is the role assigned to curvature singularities in some recent and influential theories and models of contour-based object identification and segmentation. The historical point of departure is Attneave's (1954) classic paper, in which he argued that information about shape is not distributed uniformly along the contour but is concentrated in curvature extrema. He demonstrated this by asking people to mark salient points (SPs) along the contour of a random shape and by creating a straight-line version of a sleeping cat by connecting the visually salient curvature extrema with straight lines. We saw the potential of developing special stimulus sets to test these and related ideas more thoroughly and systematically.

The research program in which these issues are being addressed currently consists of five major steps. First, we developed contour stimuli derived from line drawings of natural objects. In doing so, we have also made silhouette versions that may be useful in their own right. Second, we designed a study in which we asked people to mark SPs along the contour to test Attneave's (1954) intuition that they would pick curvature extrema. In addition, this saliency study allowed us to use normative data rather than a single individual's intuition to select SPs to create other stimuli for other purposes, such as straight-line versions analogous to Attneave's sleeping cat. So, the third step was to develop a full set of straightline versions derived from the same set of line drawings, to follow up on Attneave's demonstration in a much more systematic way, for many more stimuli and with the appropriate control conditions. Fourth, we created fragmented versions derived from the same set, as a way to cross-validate the finding that salient curvature extrema were indeed most important for identification, according to the straight-line study. Another reason was that other studies with fragmented line drawings did not have this possibility of accurate control of the location, length, and curvature of the fragments, as we had when we started from contour stimuli with known curvature values along the contour. Finally, using a subset of our contour stimuli with known identifiability, we could examine how people segment natural shapes derived from everyday objects, testing the role of curvature minima while exploring other influences, such as collinearity of contour segments, parallelism or symmetry of resulting shape segments, and even cognitive influences derived from object knowledge. In our own work, we have addressed a number of questions with these stimulus sets, but there are many more that can be asked; in addition, our custommade stimuli may be useful to other researchers in the domain of object recognition, picture identification, priming, and more, even with particular test groups such as children or brain-damaged patients.

\section{STUDY 1 Silhouettes, Outlines, and Contours}

To obtain contours of everyday objects with continuous curvature values, we started from the set of 260 line drawings developed by Snodgrass and Vanderwart (1980). This is undoubtedly the prime example of a standard stimulus set with published norms of name agreement, familiarity, complexity, and the like. It has been used quite frequently (with about 1,500 citations in the Web of Science database at the time of this writing) and has been highly instrumental in comparisons across studies of results on picture naming, object identification, priming, and so forth. Response times and developmental norms have since been provided as well (Cycowicz, Friedman, Rothstein, \& Snodgrass, 1997; Snodgrass \& Yuditsky, 1996), and other researchers have created colored versions as well as grayscale renderings of the Snodgrass and Vanderwart set (Rossion \& Pourtois, 2004).

The process from original line drawings to contours involved three major steps: (1) obtaining digital versions of the original line drawings, (2) creating silhouette versions, and (3) extracting the contours from the latter.

\section{Stimuli}

Photocopies of the original drawings by Snodgrass and Vanderwart (1980), as published in their journal article, were scanned into the computer. Using a standard graphics software package (Paint, which is part of Windows), we removed noise spots in the background and removed or added a few pixels here and there to enhance the contours of the line drawings. Silhouette versions of each line drawing were obtained by blackening the entire area enclosed by the picture's outline, effectively removing all interior features. The major requirement was that the outlines had to be closed (which is why we sometimes had to improve the contours). In most cases, a line drawing could be modified into a silhouette only by filling several smaller areas. This happened when a region 
within the whole shape also had borders that were closed (e.g., in the grapes [No. 109]; in the pumpkin [No. 181]; and in the tiger [No. 233]).

We also filled those parts of the background that were enclosed by the external contour of an object. This happened when an object contained concave parts or empty areas (e.g., the wheels of the baby carriage [No. 13] or the bicycle [No. 27] and the holes in the button [No. 41] or the chain [No. 52]). It also occurred when one part of an object partially occluded another (which happened frequently with the legs of several animals - e.g., Nos. $28,43,49,73$, and 108). Because we realized that this procedure would make many objects more difficult to recognize than they would otherwise have been, we modified some of the line drawings, especially of the animals (e.g., by placing the legs a little bit further apart). Other changes to the original line drawings were needed when an object part had only minimal area (e.g., the string attached to the balloon [No. 15] and the thread in the spinning wheel [No. 213] or the spool [No. 214]) or had a detached part (e.g., the slice of bread [No. 36] and the airflow around the top [No. 238]). Our main interest was not in the absolute levels of recognition for the silhouettes as such, but in a comparison with the outline contours.

Outline versions were created by extracting the external contours from the silhouettes (i.e., after the modifi- cations to the original line drawings had been made). For this, we made use of software tools developed at the Department of Electronics at the University of Leuven (ESAT-PSI-VISICS), building on standard computer vision algorithms for edge detection and spline fitting. These procedures resulted in smoothly curved, closed contours, which were then made discrete again for presentation on standard CRT screens. As a result, all contours were series of $x, y$ coordinates with their own local curvature values. These curvature values can be plotted in so-called curvature graphs, and curvature singularities can then be detected and selected (a software tool for this can be made available; see Table 3, A.1).

Some examples of these different versions are shown in Figure 1. We have reported results in conference presentations (Ploeger, Wagemans, Beckers, \& Vanroose, 1998; Wagemans, Notebaert, Beckers, \& Vanroose, 1997), and a full paper in which this study will be presented in more detail is under way (Wagemans et al., 2004), but we have already made these stimulus sets available on our Web site (see Table 1, A.1 and A.2).

\section{Overview of Other Major \\ Methodological Aspects}

Because in most of our studies we wanted to use specific variations of the contour versions (limiting the

Silhouette with some modifications

Figure 1. Examples of the original line drawings (Snodgrass \& Vanderwart, 1980), the silhouette versions with some modifications, and the resulting spline-fitted outlines used to derive the smoothly curved, closed contour versions. 
Table 1

Stimulus Sets

\begin{tabular}{|c|c|}
\hline Name & Link: http://www.psy.kuleuven.ac.be/ winterj/Stimuli/... \\
\hline \multicolumn{2}{|l|}{ A. Silhouettes and outlines } \\
\hline A. $1=$ Silhouettes & ... Outlines/Silhouettes BMP.zip \\
\hline \multirow[t]{2}{*}{ A. $2=$ Outlines } & ... Outlines/Outlines DIF.zip \\
\hline & ... Outlines/Outlines BMP.zip \\
\hline \multirow{2}{*}{ A. $3=$ Smoothed outlines } & ... Outlines/Smoothed DIF.zip \\
\hline & ... Outlines/Smoothed BMP.zip \\
\hline \multicolumn{2}{|l|}{ B. Straight-line versions } \\
\hline B. $1=$ Using singularities 1 extrema/lobe & $\begin{array}{l}\text {... Straight Lines/1ELOB DIF.zip } \\
\ldots \text { Straight Lines/1ELOB BMP.zip }\end{array}$ \\
\hline \multirow[t]{2}{*}{ B. $2=$ Using singularities nr equal to $\mathrm{SP}$} & .... Straight Lines/Count DIF.zip \\
\hline & ... Straight Lines/Count BMP.zip \\
\hline \multirow[t]{2}{*}{ B. $3=$ Using MP/SP marking $100 \%$} & ... Straight Lines/Salient Points DIF.zip \\
\hline & ... Straight Lines/Salient Points BMP.zip \\
\hline \multirow{2}{*}{ B. $4=$ Using MP/SP marking $75 \%$} & ... Straight Lines/Salient Points 75 DIF.zip \\
\hline & ... Straight Lines/Salient Points 75 BMP.zip \\
\hline B.5 = Tangent to MP & $\begin{array}{l}\text {... Straight Lines/Straight Line (M Tangent) DIF.zip } \\
\text {.. Straight Lines/Straight Line (M Tangent) BMP.zip }\end{array}$ \\
\hline \multicolumn{2}{|l|}{ C. Fragmented versions } \\
\hline \multirow[t]{2}{*}{ C.1 = Around MP/SP } & ... Fragments/Fragments DIF.zip \\
\hline & ... Fragments/Fragments BMP.zip \\
\hline \multirow[t]{2}{*}{ C. $2=$ Around MP/SP from straight-line versions } & ... Fragments/Fragments SL Around DIF.zip \\
\hline & ... Fragments/Fragments SL Around BMP.zip \\
\hline \multirow[t]{2}{*}{ C. $3=$ From SP from straight-line versions } & ... Fragments/Fragments SL From SP DIF.zip \\
\hline & ... Fragments/Fragments SL From SP BMP.zip \\
\hline \multirow[t]{2}{*}{ C. $4=$ To SP from straight-line versions } & ... Fragments/Fragments SL To SP DIF.zip \\
\hline & ... Fragments/Fragments SL To SP BMP.zip \\
\hline
\end{tabular}

Note - DIF is tabulated ASCII format, BMP is bitmap format. SP, salient point; MP, midpoint.

available information even further), we needed to verify whether the silhouette and contour versions could still be identified. For this reason, we asked a large group of first-year psychology students from the University of Leuven (who are expected to participate as part of a course requirement) to try to identify these stimuli, presented for $5 \mathrm{sec}$ each (at 3 -sec intervals). The participants ( $N=$ 356,74 males and 282 females) had an average age of 19.2 years. They were all native Dutch speakers, but other studies providing naming data for the Snodgrass and Vanderwart (1980) pictures in other language groups can be used to support cross-validation. Norms for picture naming using the Snodgrass and Vanderwart stimuli (sometimes combined with others) have been obtained for several languages, including Spanish (see, e.g., Sanfeliu \& Fernandez, 1996), French (see, e.g., Alario \& Ferrand, 1999), and Dutch, using participants from The Netherlands (Van Schagen, Tamsma, Bruggemann, Jackson, \& Michon, 1983 ) as well as from Flanders, the Dutch-speaking part of Belgium (Martein, 1995). In general, these studies have shown very little significant difference between language groups (for reviews, see Dell'Acqua, Lotto, \& Job, 2000, and Kremin et al., 2003).

The participants were tested in groups of about 90 in a large auditorium with a central projection system. Silhouettes and contours were shown to different groups of participants. The complete set of 260 pictures was divided into two subsets of 130 pictures drawn randomly from the original set. Thus, we organized four sessions: two for the silhouettes (with 85 and 88 participants) and two for the contours (with 94 and 89 participants). The subsets were the same for silhouettes and contours, and we also used the same random presentation order for both versions to prevent any differences between them from possibly being an accidental result of the particular stimulus sequence. Each session, including the instructions, lasted about $35 \mathrm{~min}$.

We explained to the participants that they would be shown silhouettes (or contours) of everyday objects that were created by modifying original line drawings. We also explained that this procedure would make some of the pictures virtually impossible to recognize but that we insisted that they do as well as they could. They were asked to give the most common name that best described the object presented in the picture; they were explicitly told to avoid overly general words (such as thing or ani$\mathrm{mal}$ ), words that were intended to be funny (such as inkblot), and very lengthy descriptions. They were allowed instead to indicate on their response sheets that they had failed to recognize a picture by putting a dash beside the picture stimulus number.

\section{Norms}

We used two criteria to score the responses as correct or incorrect. By the more stringent one, a response was counted as correct only when exactly the same name was given as that listed by Snodgrass and Vanderwart (1980). By the more liberal one, synonyms and dialect names 
that clearly indicated the same concept were also considered correct. However, slightly related names were not allowed (e.g., "dog" was an unacceptable response to the picture of the fox, "fly" was unacceptable for that of the bee, etc.). The participants correctly identified about half of the objects in the two versions using the samename criterion $(53.3 \%$ for silhouettes and $51.9 \%$ for contours; $S D=38.6 \%$ and $38.7 \%$, respectively) and about $10 \%$ more using the same-concept criterion $(64.7 \%$ and $61.8 \%$, respectively; $S D=39.8 \%$ and $39.6 \%$, respectively). Identification rates per stimulus varied between $0 \%$ and $100 \%$, with a large number of intermediate values (see Figure 2 for examples).

Although the naming task was performed with Dutchspeaking participants, the norms are probably useful for research in other languages as well, because the perceptual aspects of these performance levels are more interesting than the psycholinguistic ones. Indeed, it is interesting to examine why certain objects can no longer be identified in silhouette or contour versions (e.g., diagnostic texture pattern of the surface, such as that of the leopard [No. 136] or the zebra [No. 260]; distorted 3-D structure, as that of the ashtray [No. 10] or the train [No. 240]; filled-in background elements, as those of the button [No. 41] or the ladder [No. 131]). We have presented these results at conferences (see, e.g., Ploeger et al., 1998; Wagemans et al., 1997), and since then silhouette versions have been used in a good number of other studies too (e.g., Hayward, 1998; Hayward, Tarr, \& Corderoy, 1999; Lloyd-Jones \& Luckhurst, 2002). Moreover, in priming studies, it may also be interesting to select stimuli with intermediate recognition levels. Using the more liberal criteria for scoring responses as correct, we have listed all the names, the scoring, and the aggregated percentage of correct naming for both silhouette and contour versions of the Snodgrass and Vanderwart (1980) pictures (see Table 2, A.1). Our norms provide a whole range of identification rates from which researchers can sample according to their needs (see Figure 2).

\section{STUDY 2 \\ Salient Points Along Object Contours}

Attneave (1954) asked his observers to mark SPs along the contour of a random shape and noted that the most frequently marked points were situated at locations where positive curvature was maximal (i.e., $M+$ ) or negative curvature was minimal (i.e., $m-$ ). Norman et al. (2001) confirmed this observation with 16 shapes of sweet potatoes and 16 participants. Making use of our contour versions of the Snodgrass and Vanderwart (1980) set, we can test this idea on more natural stimuli that participants may identify as familiar everyday objects in many cases.

\section{Overview of Other Major Methodological Aspects}

We presented all 260 contours to a large group of participants $(N=161,34$ males and 127 females). The participants were all first-year psychology undergraduates at the University of Leuven from a different cohort in comparison with those of the other studies (mean age $=$ 19.1 years). They participated in partial fulfillment of a course requirement. The stimulus set was divided into four subsets of 65 stimuli, 1 of which was chosen randomly for each participant (yielding data from about 40 participants per stimulus). The participants were instructed to look carefully at each stimulus and then identify and mark important or salient points along the contour. Using a computer mouse, the participants could move a blue diagonal cross on the computer screen freely but always positioned on the outline. The blue cross followed the mouse pointer on the screen so that the $\mathrm{Eu}-$ clidean distance between the mouse pointer and the lo-

\begin{tabular}{|l|c|c|c|c|c|}
\hline $\begin{array}{c}\text { Outline } \\
\text { name and } \\
\text { number }\end{array}$ & Umbrella, № 245 & Plug, № 177 & Tie, № 232 & Whistle, № 255 & Accordion, № 1 \\
\hline & &
\end{tabular}

Figure 2. Examples of contour versions with variable identification. 
Table 2

Norms and Data

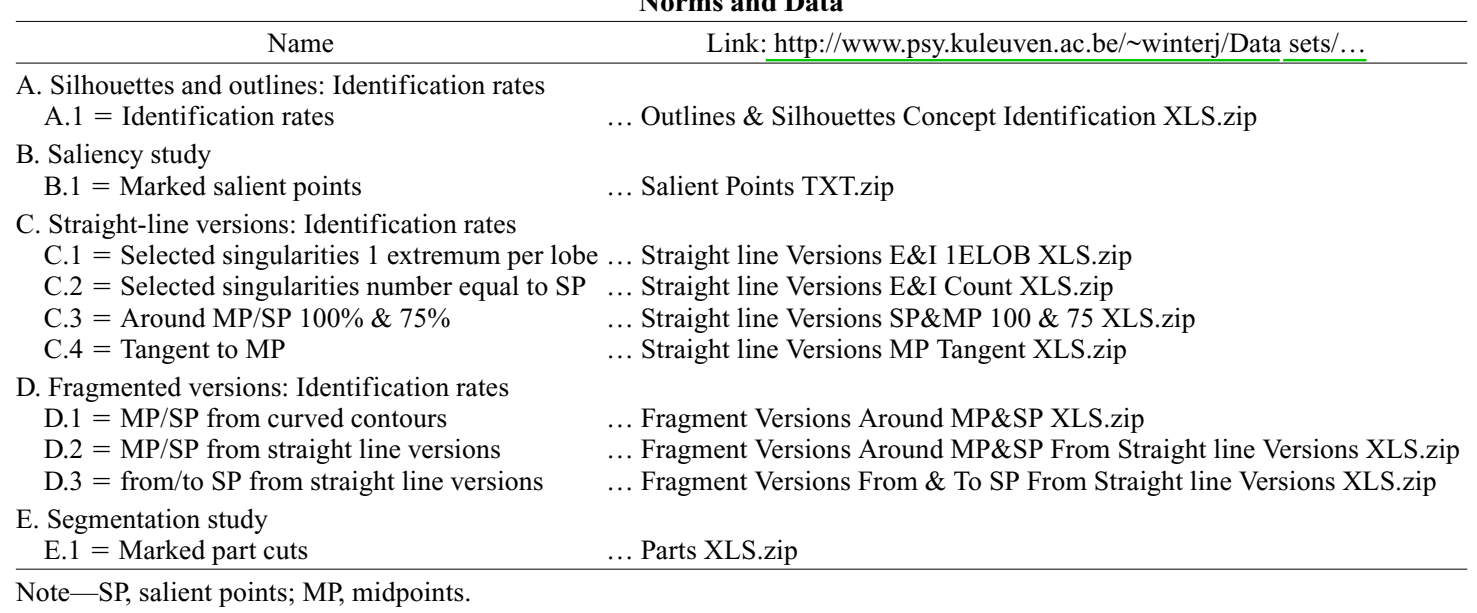

cation on the contour indicated by the blue cross was minimal. To mark a point on the contour, the participants moved the blue cross into the desired position and then clicked the left mouse button once. They had to mark at least one point, so they could not inadvertently skip any stimulus, but they could mark as many as they considered necessary. They could also correct already marked points. They could always proceed to the next stimulus at their own pace.

\section{Data}

Data from individual participants consist of contours (lists of $x, y$ coordinates) with a number of points marked as salient. The numbers of points marked along the contour differ enormously among stimuli and among participants, with a grand average of $19.8(S D=7.4)$ points. Some examples of this variation are shown in Figure 3.

Aggregated data consist of contours with the frequency of marking added in each coordinate and varying between 0 (when none of the participants marked a given point) and the number of participants for that stimulus (when all the participants marked a given point). These frequency values can be plotted along with the curvature graph of each contour (see Figure 4, black and gray lines, respectively). It appears that the participants marked about $50 \%$ of all mathematically defined curvature extrema against only about $5 \%$ of the curvature inflections. As one can imagine in light of such data, there is considerable variability, which is due partly to shape variation and partly to uncertainty or even error on the part of the participants. To deal with this variability, we have developed several software tools that allowed us to reduce this large number of data to reasonable proportions, to remove unwanted variation, and to extract the major factors underlying them.

\section{Software Tools}

Selection of salient points. We have developed a tool to aggregate marked point data, to smooth the marking frequency, and to apply a cutoff threshold to select marked points (see Table 3, B.1). This interactive tool allows the user to change all the relevant parameters, and their effects are represented visually in Figure 5. The major steps in this procedure are described in the following paragraphs.

First, the marking frequency of each point along the contour of each stimulus was determined by aggregating across all participants who had received the stimulus. These raw frequencies were then smoothed as follows. The smoothing technique was used to calculate for each point $p$ the weighted average of the frequency of point $p$ and of points in the neighborhood of $p$. Points that are further away from point $p$ should have a smaller weight on the saliency value of point $p$. We used a Gaussian function centered on each point $p$ to reflect this decreasing weight in the two directions away from point $p$ because we assume that the noise on the localization behavior of the participants is distributed according to a Gaussian function. The width of the Gaussian curve (the smoothing parameter) is expressed by its standard deviation in image pixels and is set to five image pixels in the given example (see Figure 5). This technique is known as the moving average using Gaussian weighting.

This procedure yields a smoothed saliency value for every point on the contour. The higher this value, the larger the number of participants that marked that point (or points nearby) as an SP. A comparison of the saliency plots before and after smoothing (Figures 4 and 5, respectively) makes it clear that some error on the localization is removed by this procedure. For instance, it is now clear that both corner points on the bottom of the axe's handle (i.e., the first and last black points on the saliency graph in Figure 5) have approximately the same saliency (represented by gray blobs of more or less the same diameter in Figure 5), which was not clear from the raw frequencies per se (the diameter of the blob of the leftmost corner of the axe's handle was larger than that of the rightmost corner in Figure 4), probably because local- 


\begin{tabular}{|l|l|l|} 
Mercentile \\
number of \\
points
\end{tabular}

Figure 3. Examples from the saliency study with variability across participants (left column) and across stimuli (right column). The black dots represent marked points. By participant: The contour of the baby carriage (No. 13) with salient points marked by 7 different participants (ranked according to percentile). By salient points: Seven examples of contours with a variation of number of marked points (ranked according to percentile). From top to bottom: needle (No. 154), stove (No. 219), saw (No. 196), train (No. 240), duck (No. 81), tiger (No. 233), and fence (No. 87). The diameter of the gray circles represents the (smoothed) saliency of each marked point (aggregated across participants).

ization was somewhat more precise for the somewhat sharper (i.e., the left) corner.

A good way to select SPs would be to take only the maxima of these smoothed saliency graphs. However, because some of these maxima still had very low ab- solute values (e.g., the small local maxima for points along the almost straight lines of the axe's handle), a saliency threshold was introduced as well. This is a simple cutoff in the sense that all points below a particular saliency value are no longer considered. This parameter 

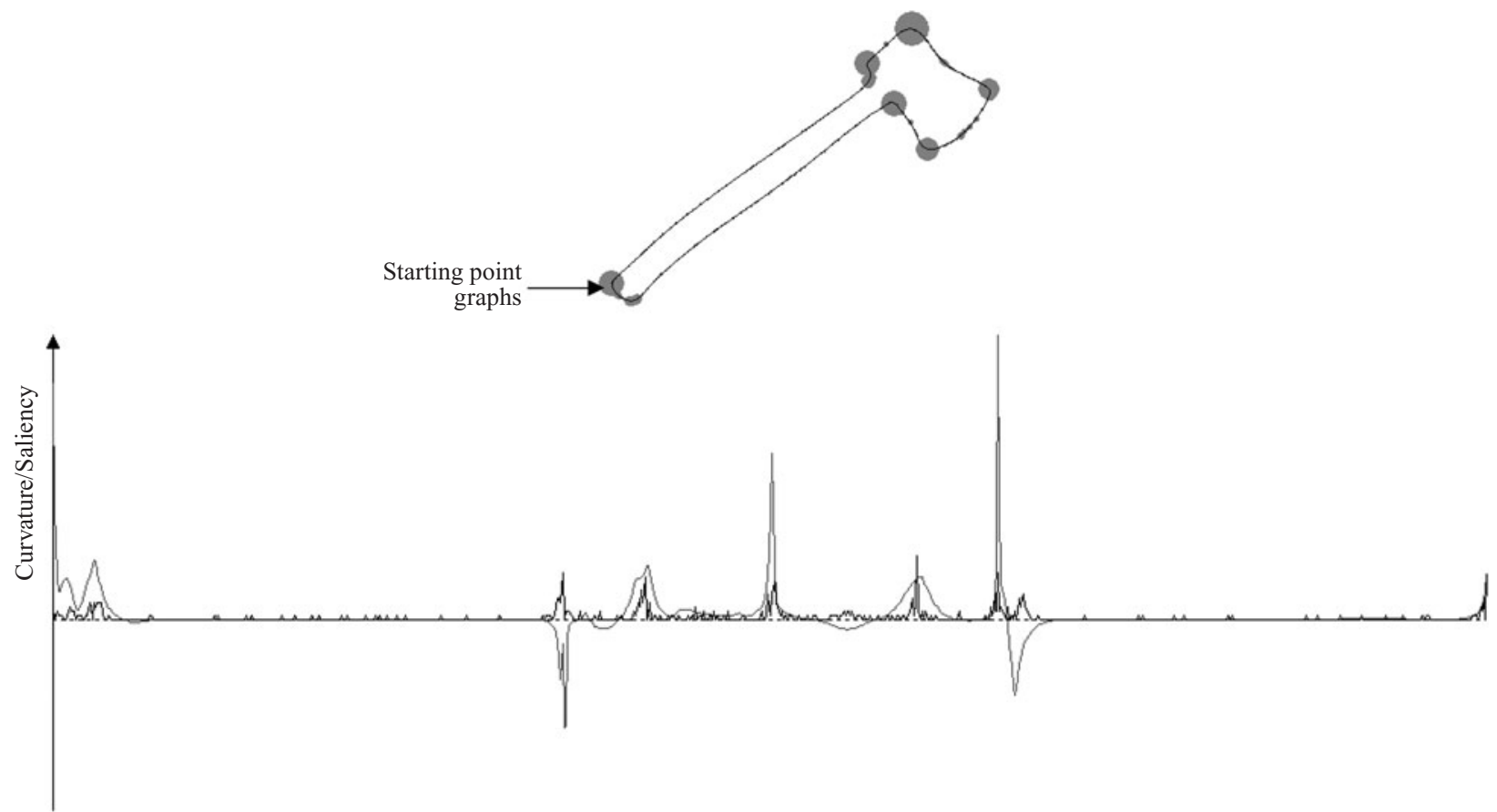

Figure 4. Upper panel: An example contour (axe, No. 12), with starting point for the curvature graph (going counterclockwise) and gray circles representing the frequency of saliency marking (larger radius = higher frequency). Lower panel: The solid dark trace is frequency plotted for each point on the contour. The gray trace plots the curvature values (i.e., the curvature graph). The dotted horizontal line is zero.

(represented by the dashed black line in Figure 5) is set to 12 in our example.

As a final step, we examined where most of the selected SPs were located along the contour. We did this by checking which special point of curvature was closest to each selected saliency maximum and how large the distance along the contour was (i.e., the summed Euclidean distances between all consecutive $x, y$ coordinates defining the contour segment). The example in Figure 5 is typical in the sense that most of the SPs were quite close to both $M+$ and $m-$.

By testing a whole range of parameter values for the smoothing parameter as well as the threshold value and by visualizing the effects as reported here (in color on the computer monitor), we could select appropriate values while minimizing unwanted side effects. The details of this process and the resulting data have been presented at a conference (De Winter, Panis, \& Wagemans, 2002) and will be reported more extensively elsewhere (De Winter \& Wagemans, 2004a). The tools will be made available on request (see Table 3, B.1); the results are already on the Web site (see Table 2, B.1).

Factors influencing saliency. We have also developed two tools to examine the factors that could have influenced the saliency of a marked point (e.g., curvature value and

Table 3

Software Tools

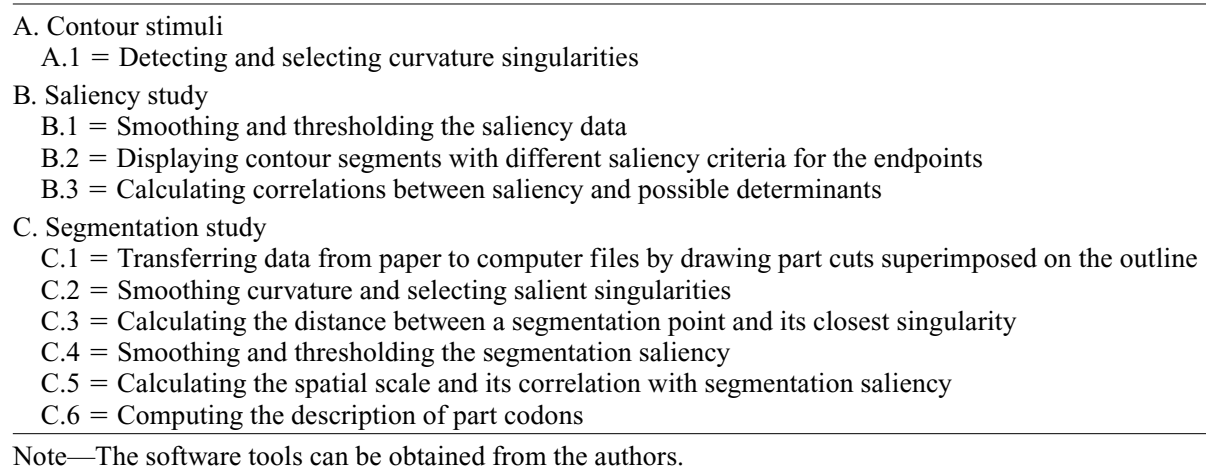



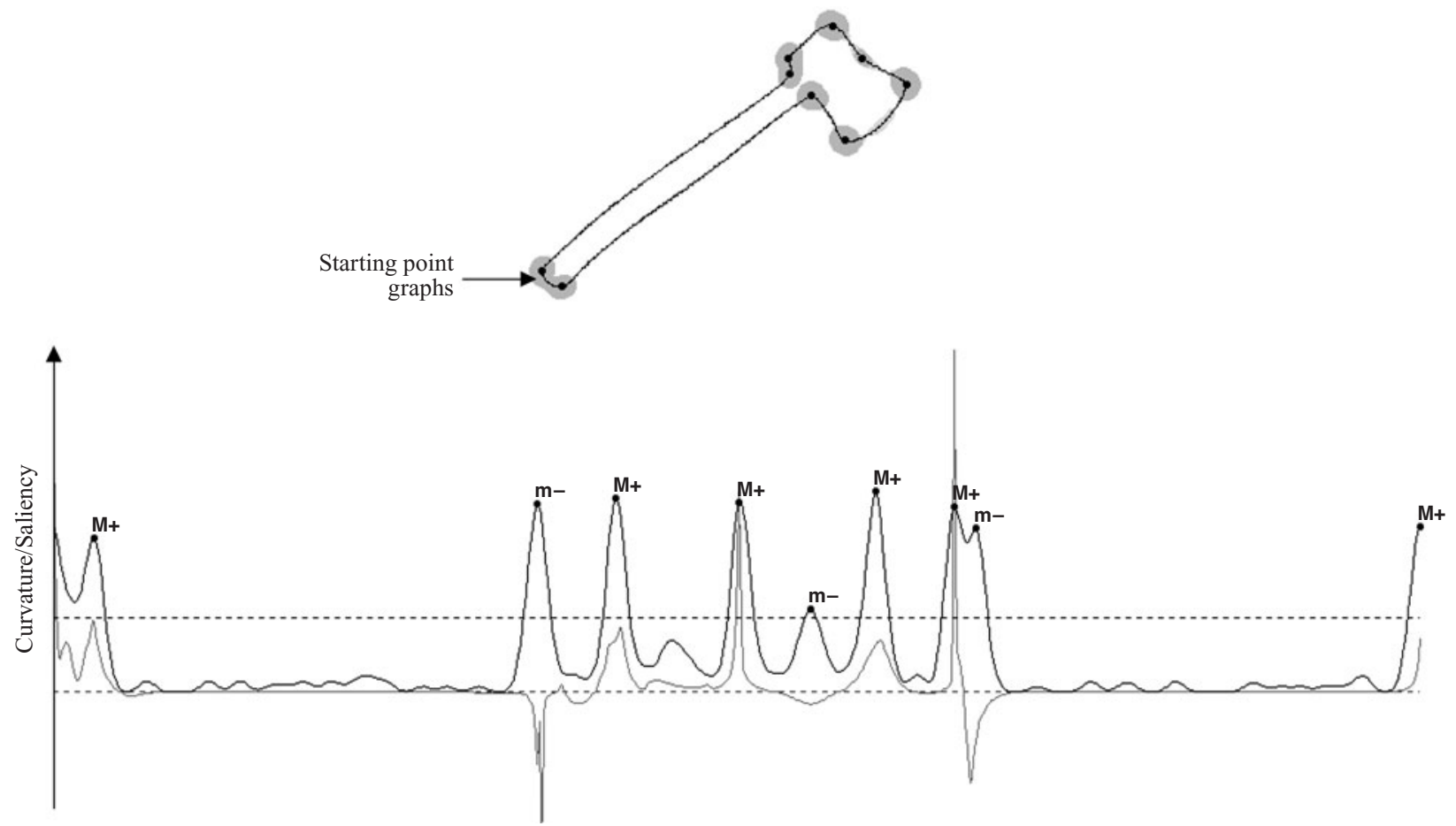

Figure 5. Upper panel: The same example contour as in Figure 4 but with smoothed saliency. The darker gray blobs have smoothed saliency values above threshold, and the lighter ones have smoothed saliency values below threshold. Lower panel: Same as in Figure 4, but now the dotted dark line represents the saliency threshold (set at 12). In both panels, black dots represent the selected saliency maxima; in the lower panel, the nearest mathematically determined curvature singularities are indicated. As one can see, the most salient points are often located very close to strong extrema (high $M+$ or deep $m-$ ).

strength of the protrusion measured in different ways). The results of these extensive analyses will be reported elsewhere (De Winter \& Wagemans, 2004a), but the tools have already been made available (see Table 3, B.2 and B.3).

The first tool (Table 3, B.2) allows us to visualize the local neighborhoods around the marked points. This tool marks contour segments using different criteria, which makes it easier to search visually for possibly important factors for a whole range of contours. To be more specific, a contour segment is defined by selecting three points: the marked point itself and two neighboring points (one at each side). Three criteria for selecting neighboring points were tested: the nearest inflection points at each side, the previous and next marked points, and the midpoints (MPs) between the current and neighboring marked points. In addition, different criteria for saliency could be tested simultaneously.

The second tool (Table 3, B.3) allows quantification of the hypothesized factors determining saliency (e.g., different parameterizations of the strength of a protrusion or the sharpness of angles) and is used to calculate correlations between these factors and the saliency values. Again, the results of these procedures can be visualized to support interactive usage. For example, to examine the sharpness of a peak (protruding or intruding from the rest of the shape) as a factor determining the saliency of a selected point, we proceeded as follows. First, we determined angle $\alpha$ between the two line segments starting at the SP, one to each of both neighboring points, and we then drew a separation line dividing angle $\alpha$ in two. This separation line was then used to align and compare all the segments of a particular saliency. In particular, the whole range of saliency values was normalized and then divided into six bins (i.e., from the weakest $1 / 6$ th to the strongest $1 / 6$ th). All contour segments (defined by the three contour points and the separation line) were now aligned (by superimposing all the separation lines) within each saliency bin and visually inspected. In this way, we found that the most salient points all belonged to contour segments with very sharp peaks (in the sense of neighboring contour segments separated by a small $\alpha$ ), whereas the length of the neighboring contour segments did not seem to matter much. This tool also allows correlations to be calculated in batch for a whole range of contour segments using different selection criteria and parameter values.

\section{STUDY 3}

\section{Straight-Line Versions}

Attneave (1954) started from a line drawing of a sleeping cat and then extracted the curvature extrema and connected them by straight lines. His paper does not contain 
any further information on how the curvature extrema were selected or how the required number of lines was determined. We suspect that Attneave relied on his excellent visual intuitions for this selection. Starting from our contours derived from line drawings of everyday objects, with their known curvature values, we can use a more principled account. This allows a better controlled, larger scale study of the effect on identifiability of removing curvature from the contours of everyday objects.

\section{Stimuli}

Selecting curvature extrema is not a trivial issue because the curvature values sometimes yield spurious local extrema that are not salient at all (see Figure 4). We have dealt with this problem in two ways. In one variant of this study, we started from the mathematically defined curvature extrema and selected only the strongest ones to be connected by straight lines. In another variant, we started from the SPs (as marked by the participants in the saliency study summarized in the previous section) and selected the strongest ones to be connected by straight lines. In the study with mathematically based straightline versions, the number of points to be selected followed the number of lobes in the curvature graph of each stimulus (i.e., the segment of the curve with a uniform sign of curvature), and within each lobe the highest peak was taken when curvature was positive and the lowest peak was taken when curvature was negative. For comparison with the saliency-based straight-line versions, we also tested a condition in which the number of selected points was equated for each stimulus as much as possible. In the saliency-based study, we selected 100\% of the most salient points (at a certain threshold) in one condition and only $75 \%$ in another condition.

Attneave (1954) did not have a control condition to support his conclusion about the role of curvature extrema. Lowe (1985) and Biederman (1988) have since shown that this is important: Connecting the points midway between Attneave's selected endpoints by straight lines also produces an easily recognizable sleeping cat; hence, extrema appear not to be special at all (see also Kennedy \& Domander, 1985). We therefore decided to use systematically selected control conditions for comparison. Because there are good reasons to expect inflections to be important for shape encoding too (Koenderink \& van Doorn, 1982), we decided to use these as starting points for comparison with the curvature extrema. Moreover, we used MPs halfway between the selected SPs to create control shapes, because these points are located as far as possible from the supposedly critically important points.

Finally, we created a specific version of straight-line stimuli, starting from MPs but keeping them from becoming spurious corner points (which is the natural consequence of their being connected by straight lines starting from and ending at the MPs) by fitting a tangent line through each of the MPs and then creating new corner points where neighboring tangent lines intersect. These versions are called midpoint tangents (MPTs). Some of these MPT versions were problematic because the intersections created " $\mathrm{X}$ " crossings or the intersection points were sticking out too far; these were eliminated from the stimulus set (yielding 142 remaining stimuli).

The details of this study with all the different straightline versions are reported elsewhere (De Winter, Panis, \&

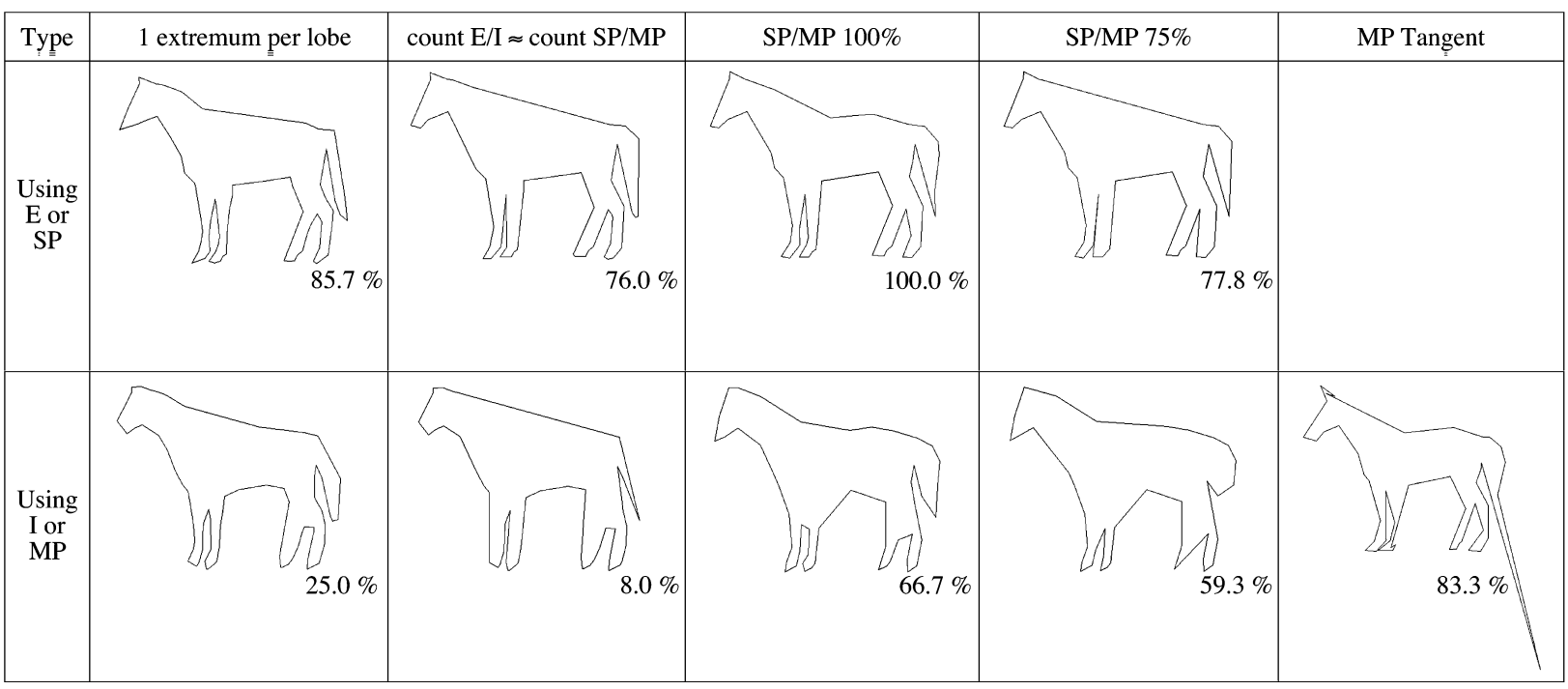

Figure 6. Examples of straight-line variants of a horse (No. 121) generated by connecting different special points with straight lines. In the first row, starting from extrema and salient points (from left to right), E1, E2, SP100, and SP75; in the second row, the corresponding conditions starting from inflections and midpoints (MPs), I1, I2, MP100, and MP75. The final condition is MPT, where straight lines are drawn through the tangents of all the MPs between salient points (see text). The identification rate for each version is given below each stimulus. 
Wagemans, 2004). Results have already been presented at conferences (Wagemans, De Winter, \& Panis, 2002).

To summarize, we have the following straight-line versions of our complete Snodgrass and Vanderwart (1980) contour set (see Table 1B): In the mathematically based experiments, E1 conditions use 1 extremum per lobe, I1 conditions use the corresponding inflections between all the selected extrema, E2 conditions use a number of extrema comparable to the number of those used in the saliency-based study, and 12 conditions use the corresponding inflections. In the saliency-based experiments, SP100 conditions use all SPs (above a certain threshold), MP100 conditions use the corresponding MPs between these SPs, SP75 conditions use only 75\% of the most SPs, MP75 conditions use the corresponding MPs between these SPs, and MPT conditions are created by drawing a tangent line through the corresponding MPs between these SPs. Examples are shown in Figure 6.

\section{Overview of Other Major Methodological Aspects}

We did not present the straight-line versions derived from the contour stimuli that were virtually impossible to identify ( $<20 \%$ correct concepts as derived from the previously described norms). Removing some extra stimuli that had very little curvature change yielded a set of 184 stimuli for this study (with an average identification rate of $83.4 \%$; $S D=22.9 \%$ ). Each stimulus was presented for a maximum of $5 \mathrm{sec}$. In all of the different variants in which straight-line versions were used, we asked another cohort of first-year psychology students from the University of Leuven to participate in partial fulfillment of a course requirement. They were tested in groups of 20-30 participants in a computer classroom equipped with 30 individual PCs with individual CRT monitors. In the first version of this study (E1 vs. I1), all 56 participants (13 males and 43 females, average age 19.3 years) received all 184 stimuli, but each stimulus was presented in only one version (either E1 or I1), so that we had 28 participants per stimulus per condition. In the second version (E2 vs. I2), the 50 participants (10 males and 40 females, average age 19.1 years) were given all of the stimuli with a similar presentation and counterbalancing procedure, which yielded 25 participants per stimulus per condition. In the saliency-based version of this study, all 108 participants (18 males and 90 females, average age 19.0 years) received the four versions (SP100, MP100, SP75, and MP75) and were assigned to the 184 stimuli in a counterbalanced fashion so that there were 27 participants per stimulus per condition. The final group of 24 participants ( 4 males and 20 females, average age 18.8 years) received all MPT versions of the 184 stimuli once.

\section{Norms and Data}

As in the previous naming study, data from individual participants consist of given names for each stimulus, which were scored as correct or incorrect (on the basis of concept) and then aggregated across participants and stimuli in each of the different conditions (see Table 2C). As we expected, identification rates varied considerably between stimuli and conditions (see Figure 6).

The participants correctly identified about half of the stimuli in the extrema-based conditions (for E1, 46.4\%, $S D=39.3 \%$; for E2, $53.4 \%, S D=36.4 \%$ ) and clearly fewer in the inflection-based conditions (for I1, 20.4\%, $S D=28.1 \%$; for $\mathrm{I} 2,14.2 \%, S D=23.7 \%$ ). A similar trend was present in the saliency-based conditions, in which SP versions were much easier to recognize than MP versions (for SP100, 68.9\%, SD = 34.1\% and for $\mathrm{SP} 75,54.7 \%, S D=37.8 \%$ vs. $34.4 \%, S D=32.9 \%$ for MP100 and $18.0 \%, S D=25.7 \%$ for MP75). Note that the identification rates of SP and MP versions were significantly higher than those of the straight-line versions connecting mathematically derived singularities (E2 and I2, respectively). Although SPs are often located near very high $M+$ or very low $m-$, this observation suggests that the saliency of the selected corner points is more important than the curvature value as such. The identification rate for MPT was $60.2 \%(S D=33.0 \%)$, which is much higher than that for MP100/75 and much closer to the identification rate of the comparable SP100 condition. This indicates that the deteriorated identification of the MP straight-line versions in comparison with that of the SP straight-line versions is due mainly to the fact that the MPs have become spurious corner points; when new corner points are introduced in locations other than the MPs whereas the average orientation of the lines passing through the MPs is more or less preserved, deteriorated recognition recovers almost completely.

These results are presented in more detail elsewhere (De Winter et al., 2004) but they are already available on the Web site (see Table 2B). In future studies, specific stimuli can be used because of their specific levels of deteriorated recognition (e.g., in priming studies), or the results can be examined further to find reasons for these specific recognition rates (e.g., stimulus-specific factors having to do with degree of contour curvature variation, number of selected endpoints).

\section{STUDY 4 Fragmented Versions}

In the context of object identification from line drawings, picture fragmentation has long been used as a technique of stimulus degradation (see, e.g., Leeper, 1935; Street, 1931). However, two developments have led to a further increase of its popularity since the late 1980s and early 1990s. First, Biederman and his co-workers have used different types of fragmented pictures to provide evidence for his recognition-by-components theory. To be specific, Biederman and Blickle (1985, cited in Biederman, 1987) showed that object recognition deteriorated very much when the object's basic components could no longer be recovered from the fragments, whereas object 
recognition was pretty well preserved when the components could still be recovered from the fragments. Biederman and Cooper (1991) tested long-term priming with different types of fragmented pictures as primes and targets, and showed that the visual component of the priming effects was situated largely at the level of the structural descriptions consisting of object components, not at the fragment or whole-object level.

Second, in analogy with the word-fragment completion task used to examine implicit memory with verbal material, picture-fragment completion has been used as a tool to study implicit memory using pictures instead of words (for a review on implicit memory, see Tulving \& Schacter, 1990). Snodgrass and Corwin (1988) made fragmented versions of a subset of the original Snodgrass and Vanderwart (1980) set, with eight fragmentation levels increasing from very few and small fragments to complete pictures, and they determined identification thresholds for the whole series of fragmentation levels for each picture. Since then, Snodgrass has used this stimulus set in a large number of studies, testing conceptual priming in fragment completion (Hirshman, Snodgrass, Mindes, \& Feenan, 1990), the perceptual closure hypothesis (Snodgrass \& Feenan, 1990), the role of stimulus similarity (Snodgrass \& Hirshman, 1994), and the sensory match effect (Snodgrass, Hirshman, \& Fan, 1996), as well as electrophysiological correlates (Viggiano \& Kutas, 2000) and developmental trajectories (Cycowicz, Friedman, Snodgrass, \& Rothstein, 2000).

Using our contour versions of the Snodgrass and Vanderwart (1980) set with the curvature values for all points defining the complete contour, we can introduce different types of fragmentation with strict control on the lengths and locations of the fragments. For example, we can test Attneave's (1954) hypothesis about the role of curvature extrema using fragmented versions rather than straight-line versions - namely, by having an equal percentage of the contour distributed differently along the contour, located either on curvature extrema (or SPs) or on inflections (or MPs). Because we are convinced that the parametric control on fragment length and location in our fragmented versions has certain advantages over the previously developed stimulus sets, we believe it is useful to share our stimulus sets, norms and data, and software tools with other researchers.

\section{Stimuli}

In our earliest published study with these stimuli (Wagemans, Notebaert, \& Boucart, 1998), we used a paradigm in which we could measure identification rates continuously by allowing the participants to build up the contour gradually (adding a percentage of the complete contour by pressing the space bar on the keyboard of a PC) around the $M+, m-$, or $I$ of curvature. Because this leads to a different number of fragments in each condition, we have compared only two fragmentation conditions in later studies (e.g., Wagemans, Panis, De Winter, \& Op de Beeck, 2001), using fragments located around both $M+$ and $m-$ in one condition and fragments located around $I$ in another. Finally, relying on the data from the largescale saliency study described earlier, we have also developed stimulus sets in which the fragments could be located on either highly salient points or points midway between the SPs (i.e., at the MPs). Although we can provide stimuli using the whole range of fragmentation levels from a little more than $0 \%$ to almost $100 \%$ of the con-

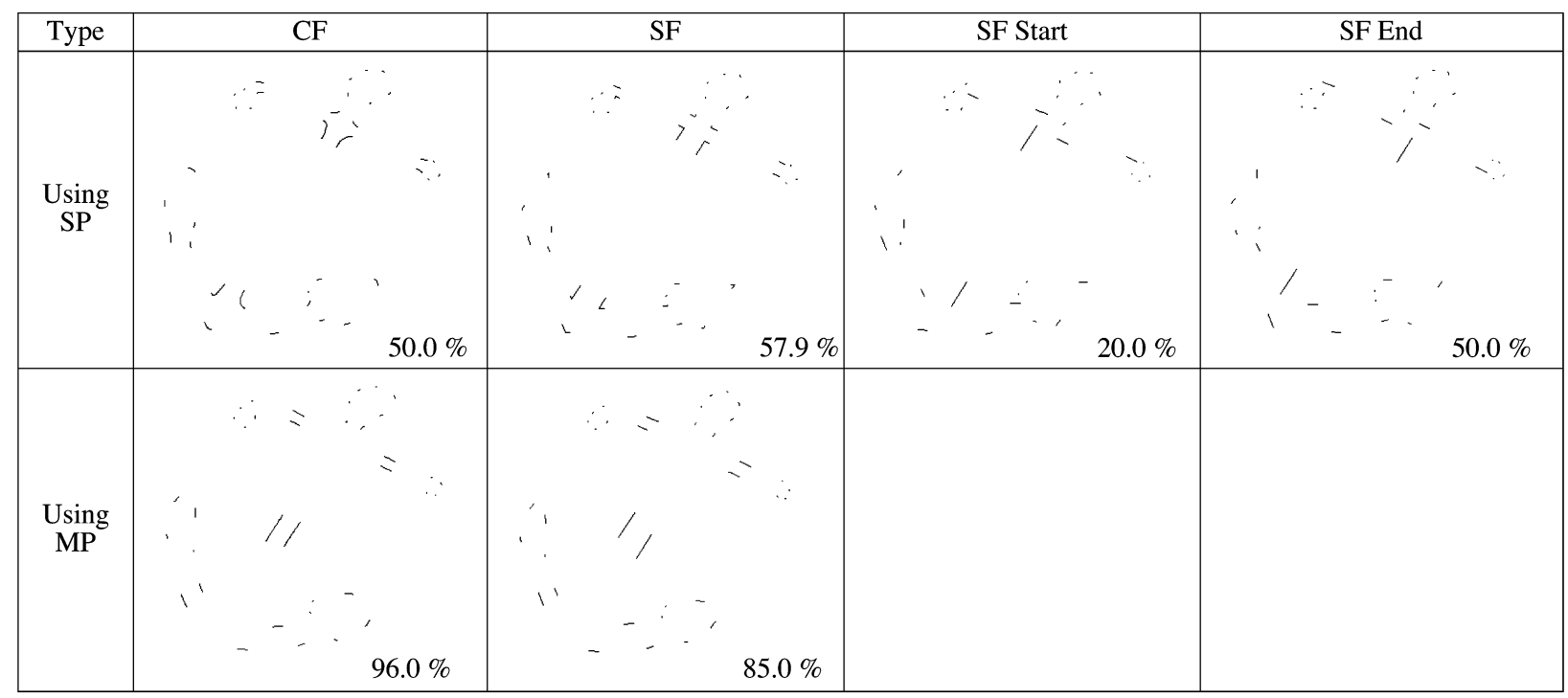

Figure 7. Examples of fragmented versions of an anchor (No. 4) using $15 \%$ of the contour, generated using different special points (SPs) and different outline versions (see text). Identification rate for each version is given in the lower right corner below each stimulus. MP, midpoint; CF, contour-based fragmented versions; SF, straight-line-based fragmented versions. 
tour, we have studied the range between $15 \%$ and $30 \%$ in most detail, because below that range identification rates are usually too low and above that range recognition becomes too easy (see Figure 7 for examples). Very much to our surprise, fragmented versions with the fragments located at the MPs were systematically easier to identify than fragmented versions with an equal fraction of the contour presented but with the fragments located at the SPs (Panis, De Winter, \& Wagemans, 2002). This result goes completely against Attneave's (1954) hypothesis in the sense that this time (in contrast with the results from the study with the straight-line versions summarized above) SPs (very often the perceptually strongest curvature extrema) appear less useful for recognition than MPs (very often quite far from salient curvature extrema).

To better understand the source of this conflicting result, we have developed other stimulus sets too, taking fragments from the straight-line versions rather than from the curved-contour versions, thus effectively maintaining the same average fragment locations and orientations but removing all curvature from the fragments. More specifically, in one condition we started from the SP-straightline versions and used $15 \%, 20 \%$, and $25 \%$ of the total line length, whereas in another condition we used similar fragmentation levels starting from the MP-straightline versions. To equate average length of fragments for SP and MP conditions and to eliminate local segmentation cues in the SP conditions, we also made versions in which the straight-line fragments started from SPs or ended in them. The details of this complete fragmentation study is reported elsewhere (Panis, De Winter, \& Wagemans, 2004), but intermediate results have already been presented at conferences (Panis et al., 2002).

To summarize, we have the following fragmented versions derived from our complete Snodgrass and Vanderwart (1980) contour set (see Table 1C): Using the splinefitted, smoothly curved contours, CF_SPs have curved fragments on SPs and CF_MPs have curved fragments on the MPs between these SPs; using straight-line versions, SF_SPs have straight-line fragments on SPs, SF_MP have straight-line fragments on the MPs between these SPs, SF_SP_start have straight-line fragments starting at SPs, and SF_SP_end have straight-line fragments ending in SPs. Examples are shown in Figure 7.

\section{Overview of Other Major Methodological Aspects}

As in the study with the straight-line versions, we did not present the fragmented versions derived from the contour stimuli that were virtually impossible to identify $(<20 \%$ correct concept as derived from the norms from the first study). Removal of some extra stimuli that had very little curvature change yielded a set of 188 stimuli for this study (with an average identification rate of $82.5 \%, S D=23.7 \%$ ). Each stimulus was presented for a maximum of $5 \mathrm{sec}$. In all of the different variants in which straight-line versions were used, we tested firstyear psychology students from the University of Leuven, who had not participated in any of the other studies and who participated in partial fulfillment of a course requirement. They were tested in groups of 20-30, with individual PCs. In the first version of this study, in which contour-based fragments (CFs) were used, all 202 participants (40 male and 162 female, average age 18.9 years) received all 188 stimuli, but each individual stimulus was presented only once, in only one of the eight versions (CFs with SPs or MPs, using $15 \%, 20 \%, 25 \%$, or $30 \%$ of the contour). Stimuli were assigned to conditions in such a way that we had data from about 25 participants per stimulus per condition. In the second version of this study, in which straight-line-based fragments (SFs) were used, the 232 participants (56 males and 176 females, average age 19.3 years) received only half of the total stimulus set (i.e., 94 stimuli) with the stimuli assigned in a counterbalanced fashion to the six conditions (SF_SP and SF_MP using $15 \%, 20 \%$, or $25 \%$ of the contour), yielding about 19 participants per stimulus per condition. The final group of 119 participants ( 17 males and $102 \mathrm{fe}-$ males, average age 19.4 years) also received 94 stimuli each, divided evenly over all six conditions (SF_SP_start and SF_SP_end, using $15 \%, 20 \%$, or $25 \%$ of the contour) in a counterbalanced fashion so that each stimulus was seen by each participant only once, yielding about 10 participants per stimulus per condition.

\section{Norms and Data}

As in the previous naming studies, data from individual participants consist of given names for each stimulus, which were scored as correct or incorrect (on the basis of

Table 4

Identification Rates (\% Correct) and Standard Deviations in the Study With the Fragmented Versions

\begin{tabular}{|c|c|c|c|c|c|c|c|c|}
\hline \multirow{2}{*}{$\begin{array}{c}\text { Type of } \\
\text { Fragmented } \\
\text { Version }\end{array}$} & \multicolumn{2}{|c|}{$15 \%$} & \multicolumn{2}{|c|}{$20 \%$} & \multicolumn{2}{|c|}{$25 \%$} & \multicolumn{2}{|c|}{$30 \%$} \\
\hline & $\%$ Correct & $S D$ & $\%$ Correct & $S D$ & $\%$ Correct & $S D$ & $\%$ Correct & $S D$ \\
\hline CF_SP & 45.3 & 33.7 & 52.3 & 35.4 & 58.1 & 34.8 & 62.8 & 34.2 \\
\hline CF_MP & 52.7 & 34.8 & 59.9 & 34.2 & 64.7 & 34.3 & 67.4 & 33.6 \\
\hline SF_SP & 48.9 & 34.6 & 54.2 & 35.0 & 59.8 & 35.6 & & \\
\hline SF_MP & 50.3 & 35.6 & 57.0 & 34.6 & 61.2 & 35.1 & & \\
\hline SF_SP_start & 41.5 & 35.1 & 48.6 & 35.4 & 51.6 & 35.5 & & \\
\hline SF_SP_end & 43.7 & 35.2 & 49.7 & 36.2 & 53.5 & 35.6 & & \\
\hline
\end{tabular}

Note-SP, salient points; MP, midpoints; CF, curved fragmented versions; SF, straight-line fragmented versions. 
concept) and then aggregated across participants and stimuli in each of the different conditions (see Table 2D). As we expected, identification rates varied considerably between stimuli and conditions (see Figure 7).

The variation across conditions is summarized in Table 4. As can be seen in the table, fragmented versions derived from smoothly curved contours (CFs) were systematically more difficult to identify when the fragments were located at SPs than when they were located at MPs (at all levels of fragmentation). This is a surprising finding in light of Attneave's (1954) hypothesis and the robust findings from the study with straight-line versions. However, this difference (of about $4.5 \%$ to about $7.5 \%$ ) disappears almost completely (to about $1 \%-3 \%$ ) when the curvature is removed from the fragments, as in the fragmented versions derived from straight-line versions (SFs). Finally, the fragmented versions in which the SFs start from or end at SPs are the most difficult to identify. It is clear that fragment curvature and perceptual grouping problems play a role in these effects. We are still doing extra analyses, for example, by correlating these recognition differences with systematic stimulus differences in factors such as number, length, and curvature of fragments, with distribution along the contour either being more or less random or creating locally parallel pairs, and so on (see Panis et al., 2004), but the stimuli and corresponding results can be made available on the Web site already (see Tables 1C and 2D).

\section{STUDY 5 \\ Segmentation Points Along Object Contours}

Since Hoffman and Richards (1984) introduced their minima rule of segmentation as a cornerstone of their theory of object recognition on the basis of parts, the topic of object segmentation has received renewed attention. For example, Biederman (1987) strongly developed two aspects in his recognition-by-components theory: the role of nonaccidental properties to support the recovery of 3-D object characteristics from 2-D image regularities and the idea that basic-level object identification can be achieved when each segmented part can be classified as one of 36 categorically distinct building blocks. Biederman (1987) did not specifically address the issue of how the parts arise perceptually in the first place; he simply relied on Hoffman and Richards's minima rule of segmentation to solve this problem. However, this theory is often insufficient to solve the segmentation problem completely. For example, it gives segmentation points but does not yield part cuts consisting of matched pairs of segmentation points. Pairs of minima are seldom defined in a unique fashion, and choices must be made when the neighborhood around a negative minimum contains multiple negative minima (and, thus, one of them must be chosen to yield a unique pair of minima) or no other minima at all (and, thus, another point must be chosen to yield a pair of segmentation points).
As a result, Hoffman and colleagues have complemented the minima rule with other factors (e.g., saliency; see Hoffman \& Singh, 1997) and rules (e.g., the shortestcut rule; see Singh, Seyranian, \& Hoffman, 1999). Rather than start from specific segmentation points, other researchers have proposed rules that yield specific types of parts, such as the largest convex patches (see Vaina \& Zlateva, 1990) or limbs and necks (see Siddiqi \& Kimia, 1995). In light of this wealth of theoretical proposals for segmentation and part-finding rules, it is rather disappointing to see that the empirical support is generally based on small numbers of test stimuli (line drawings of everyday objects or simple geometric drawings) and participants. Using our contour versions of the Snodgrass and Vanderwart (1980) set, we can provide solid benchmark data to test segmentation models on a much larger scale.

\section{Stimuli and Other Major Methodological Aspects}

We presented 88 of our contour stimuli to 201 firstyear psychology undergraduates at the University of Leuven who had not participated in any of the other studies (41 male and 160 female, average age 19.4 years). We asked them first to try to identify each object and then to segment it into its parts by drawing lines that intersect the contours in two places (i.e., defining part cuts rather than marking individual segmentation points as such). We also asked other participants to mark individual segmentation points (in fact performing 1-D contour segmentation rather than 2-D shape segmentation), but we will not dwell on that condition here (see De Winter \& Wagemans, 1999). The stimuli consisted of two lists of 44 contours, one with easy-to-recognize objects (i.e., $>70 \%$ correct concept naming according to the normative study described earlier) and one with difficult-torecognize objects (i.e., $<30 \%$ correct), but matched for visual complexity (i.e., operationalized by the number of inflections). This was done to allow us to examine the role of top-down influences on object segmentation. Each participant received only a subset of 11 easy-torecognize and 11 difficult-to-recognize stimuli. With some data trimming (because of obvious sloppiness in some participants' records), this yielded segmentations by about 40 participants per stimulus per condition.

\section{Data and Software Tools}

Data from individual participants consist of object names (which can then be classified as correct or incorrect according to the criteria described earlier) and marked part cuts for each stimulus. Across all contours, 10,773 part cuts were made, with somewhat more in the easy condition than in the difficult condition $(6,085$ vs. 4,688 , or $56.5 \%$ vs. $43.5 \%$, respectively). Across all participants, each stimulus received an average number of 122.4 part cuts $(S D=80.13)$ and across all stimuli each participant drew 68.6 part cuts $(S D=19.47)$, which means that each 
contour was segmented in three or four parts on average (i.e., 2 or 3 cuts). This large database of part cuts was analyzed extensively in an attempt to test the existing segmentation models and, perhaps, to discover new principles as well. While doing so, we developed a large number of software tools that other researchers may also find useful (see Table 3C for an overview). The results of this largescale segmentation study have been presented at a couple of conferences already (De Winter \& Wagemans, 2001, 2003) and will be reported in full detail elsewhere (De Winter \& Wagemans, 2004b). In the context of this paper, a brief overview of the database and the tools should be sufficient to assess their potential value for the larger research community. Five major steps can be distinguished.

First, the paper-and-pencil data from the participants had to be transferred onto the computer. The tool that supported this transfer procedure (see Table 3, C.1) presented a selected contour on the computer screen and allowed the user to draw a line superimposed on the contour. The locations along the contour that were intersected by this line were marked by two blue dots. When the user agreed that these corresponded nicely with the paperand-pencil markings to be entered, he or she could click the right mouse button and the $x, y$ coordinates of these segmentation points were then saved as pairs constituting a part cut. These "raw" data are made available on the Web site (see Table 2E).

Second, to examine whether the segmentation points were closer to negative minima as predicted by Hoffman and Richards's (1984) minima rule, two extra tools were needed. One tool (see Table 3, C.2) extracted the curvature singularities for all the contours and applied certain selection filters to prevent visually nonimportant singularities from affecting the subsequent analyses. For example, the absolute curvature value had to be larger than a certain threshold value, and consecutive singularities of the same type had to have sufficiently different curvature values. As before, these thresholds could be chosen appropriately by a visual inspection of their effects. Because we realized that this procedure introduces some

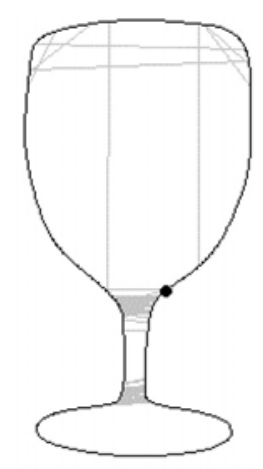

A

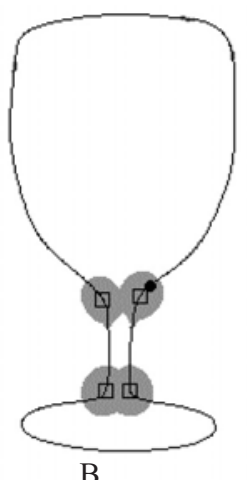

B

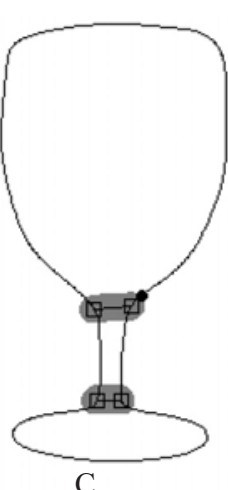

$\mathrm{C}$

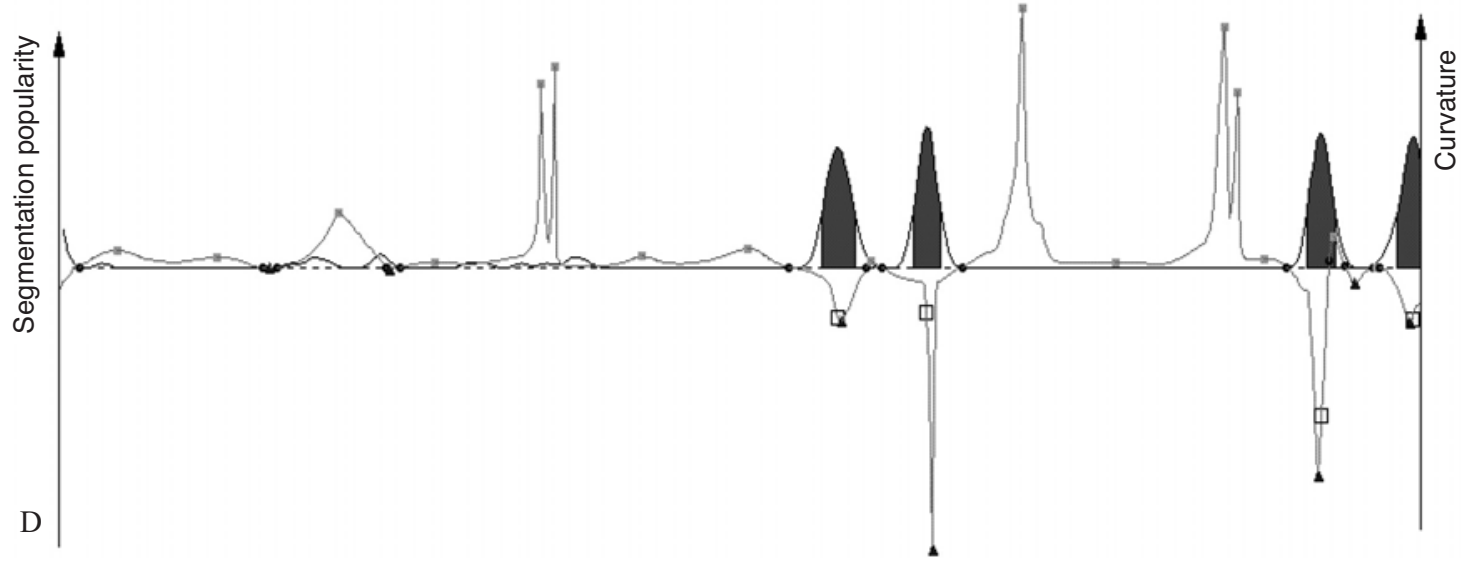

Figure 8. (A) The outline of a wineglass (No. 258) as segmented by subjects (gray lines). (B) The gray blobs represent the segmentation saliency (larger radius $=$ higher saliency). The squares represent local saliency maxima and are considered as the (anchor) segmentation points. (C) The thick dark lines represent the summarized part cuts (with a thin black line at the center). The thickness of the dark lines represents the consistency of the part cut across participants (thicker is higher). (A, B, and C) The black dot on each outline represents the starting point to trace the contour of the stimulus (counterclockwise) and plot the corresponding segmentation saliency and curvature graph (D). (D) The black line represents the saliency of segmentation points along the outline. The dark sections have a saliency above the threshold (10). The curvature graph (gray) is superimposed. Note that the anchor points (open squares) are all located near $m$ - points (small triangles). 
arbitrariness, we decided to perform the saliency study described above. Another tool (see Table 3, C.3) is used to find which curvature singularity (from the selected subset) is closest to each segmentation point in the database and calculates the distance along the contour (summed Euclidean distances between consecutive contour coordinates). It also allowed us to aggregate the results for all the segmentation points in the database. On the basis of these analyses, it was clear that $m-$ are indeed most popular as segmentation points. For example, they were the nearest neighbor in $63.3 \%$ and $68.4 \%$ of the cases in the difficult and easy conditions, respectively; $I$ and $M+$ were clearly less popular (between $12 \%$ and $19 \%$, respectively). These differences became even larger when one considers only the singularities at a maximal distance of 10 pixels from the segmentation points. The segmentation points that were very close to $m$ - also turned out to be the most popular ones (i.e., chosen by the largest number of participants).

Third, we developed a tool to apply smoothing and thresholding to the current set of marked segmentation points (see Table 3, C.4) in analogy to similar procedures for the marked points in the saliency study summarized above. Consider the example of the wineglass (No. 258) shown in Figure 8, as segmented by our participants (gray lines in Figure 8A). Saliency of individual segmentation points was determined by smoothing each point in each cut with its immediate neighbors (yielding the gray blobs shown in Figure 8B). The local maxima of this smoothed segmentation saliency were then considered as the intended segmentation points or anchor points (indicated by open squares in Figures 8B and 8C), provided that their value was over a certain threshold (again determined after visual exploration). Then, all similar part cuts were aligned on the same pair of anchor points if their segmentation points were within a certain distance along the contour. In this way, the "raw" part cuts could be aggregated to determine the saliency of part cuts (visualized by the thickness of the gray lines in Figure 8C), provided again that this part cut saliency was also above a certain threshold value. By comparing the location of the open squares to the location of the small triangles in the curvature graph in Figure 8D, it becomes clear that the most salient segmentation points were very close to negative minima. Considering the level of the part cuts rather than the individual points, the majority of the cuts (about 65\%) had at least one negative minimum, with about one third of these being even $m-/ m$ - pairs (another third used $M+$ or $I$ as the second point, and another third used just another point, not a singularity). These results thus support the minima rule of segmentation, but they also illustrate very well that other factors are playing a role too.

To deal with the important fact that structure exists on different spatial scales, Witkin (1986) developed the concept of scale space (i.e., the extraction and representation of image features such as edges and corners at a whole range of spatial scales). The fourth tool (see Table 3 , C.5) allows us to examine similar ideas for our segmentation data and curvature values. More specifically, it calculates how long a particular curvature singularity survives over increasingly larger spatial scales, from the finest spatial scales, with all sorts of tiny changes from one point to the next, to the very large scale at which curvature changes only very gradually. Figure 9 shows some of the versions of the wineglass at increasingly larger spatial scales (correcting for size changes using a procedure developed by Lowe, 1989). Differently smoothed

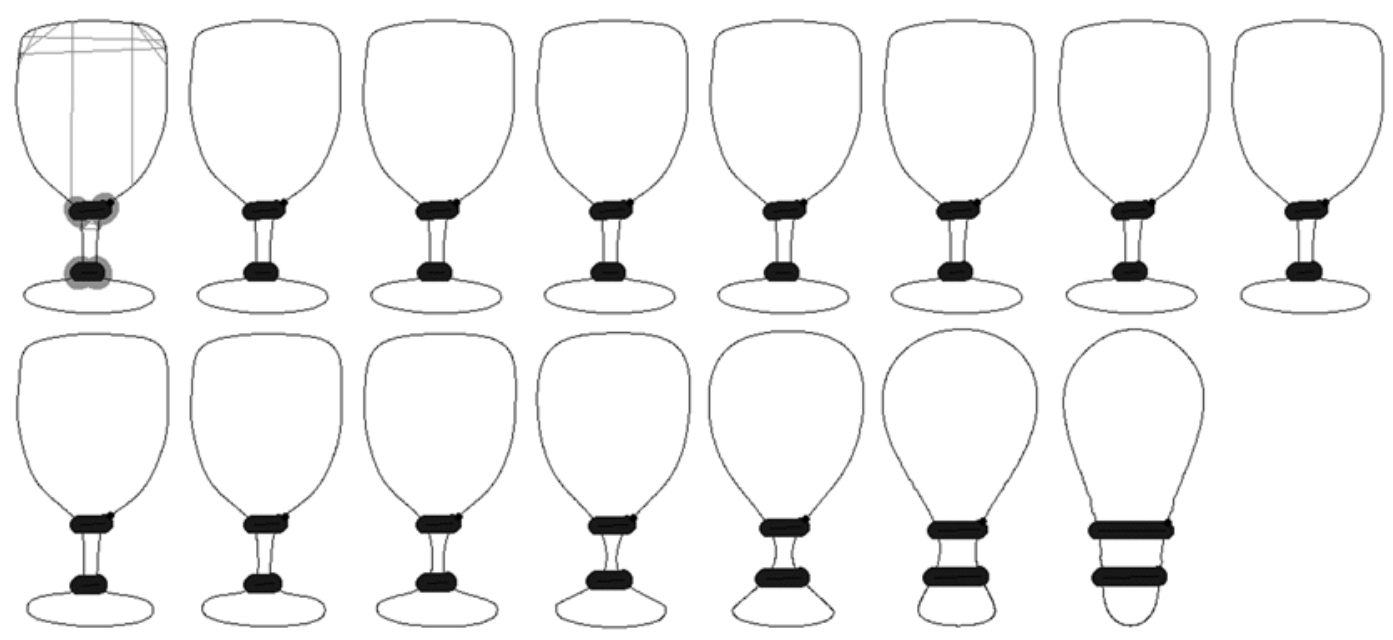

Figure 9. The outline of a wineglass (No. 258) smoothed at different spatial scales (using correction for shrinkage; Lowe, 1989). The upper left outline is the original nonsmoothed outline with the segmentation data superimposed; the others are gradually smoothed more and more. Sigma value starts at 1 and is then multiplied by $\sqrt{ } 2$ for each subsequent smoothed outline. The thick black lines are the summarized part cuts (see Figure 8C) superimposed on the smoothed outline. 
versions of many of our outline stimuli are made available on the Web site too (see Table 1, A.3). The important idea in the context of this study is that the so-called scale of a singularity (i.e., how long it survives in scale space going from fine to large spatial scales) determines its visual saliency. For the negative minima, there was a significant positive correlation between their popularity as segmentation points and their scale, as was expected ( 0.35 and 0.36 for easy- and difficult-to-recognize stimuli, respectively). For the other singularities, the correlation was negative $(-0.19$ and -0.20 , respectively, for $M+)$ or negligible $(0.01$ and 0.08 , respectively, for $I)$.

Fifth, a separate tool (see Table 3, C.6) was developed to describe the contour segments between the two points of the strongest part cuts in terms of their codon configuration (according to the codon theory developed by Richards \& Hoffman, 1985). Many different codon configurations existed in our large data set, but $m-M+m-$ was clearly the most dominant sequence, especially at the larger spatial scales.

In summary, by visually representing segmentation points and part cuts, we were able to find many instances of known part cuts from the literature (minima rule, shortest cuts, necks, limbs, etc.), but we also discovered new factors that seem to have an influence on the selection of part cuts as well (e.g., collinearity, curvilinearity, elongation, quasisymmetry, and even cognitive influences). We also realized that local factors (at the level of contour curvature) interact with more global factors (at the level of the whole shape or the shape of the resulting part) so that the previously proposed models from the literature could, in fact, be integrated into one coherent theoretical framework, along with the newly discovered factors. We are convinced that only a detailed investigation of such a large data set, which was made possible by all the different tools described above, could have produced this progress in our understanding of object segmentation into parts.

\section{GENERAL DISCUSSION AND CONCLUSION}

We have summarized the most important aspects of the stimuli, norms and data, and software tools of five studies in which aspects of contour-based object identification and segmentation have been examined. Stimuli were always derived from the very popular standard set of line drawings of everyday objects by Snodgrass and Vanderwart (1980), in the sense that we extracted the contours of the outline shapes and used contour curvature as a major variable in all of our studies. In the set of all five studies, a total of over 1,500 participants were used, producing very solid, normative identification rates of silhouettes and contours $(N=356)$, straight-line versions $(N=238)$, and fragmented versions $(N=553)$ and quite reliable benchmark data about saliency of points $(N=161)$ and object segmentation into parts $(N=201)$.
In addition, the identification rates for several different conditions within the studies in which fragmented and straight-line versions were used are based on a substantial number of participants per stimulus per condition (usually around 25). These data also have great potential for further exploration of factors influencing contourbased object identification. In this large-scale research program, we have also developed several software tools to generate stimuli and to analyze the data in nonstandard ways (doing a great deal of image processing, with which most experimental psychologists are not familiar). We can share our expertise in this area by providing assistance to other researchers who want to use similar tools in their own research (using their own stimuli and results, or ours if they wish).

Let us give some examples of recent topics of research that could benefit from our stimuli, norms and data, and software tools. In computer vision, many models have been developed to extract and process contours that need to solve the problem of contour-fragment grouping and that often use object-level knowledge (see, e.g., Bergevin \& Bubel, 2003; Elder \& Goldberg, 2001; Elder, Krupnik, \& Johnston, 2003; Jiang, 2000). These models, and similar ones developed for human visual perception (see, e.g., Feldman, 2001; Kellman, 2003), can now be tested on a much larger data set. Also in computer vision, many models have been developed for object or shape segmentation (see, e.g., Bilodeau \& Bergevin, 2002; Cronin, 2003; Dhandapani \& Kimia, 2002; Rosin, 2000) in addition to scene segmentation (which is not done with contour and shape so much as with surface texture statistics; for an extensive review, see Freixenet, Muñoz, Raba, Martí, \& Cufi, 2002). As far as we know, these models have not been tested with human benchmark data of the type we have. In cognitive neuropsychology, patients with agnosia have been tested with a small number of stimuli to study the interaction between perceptual grouping and object perception (see, e.g., Behrmann \& Kimchi, 2003; Delvenne, Seron, Coyette, \& Rossion, 2004; Giersch, Humphreys, Boucart, \& Kovacs, 2000; Piccini, Lauro-Grotto, Del Viva, \& Burr, 2003). We believe our stimulus sets have great potential for this type of studies too. Similarly, in studies of unconscious or conscious priming of object perception and picture naming, it might be interesting to make use of a set of primes with known identifiability rates across the whole range from 0 to $100 \%$. In sum, we hope that our stimuli, norms and data, and software tools will be useful to a large community of researchers from many disciplines working on a wide variety of research topics.

\section{REFERENCES}

Alario, F.-X., \& FERRAND, L. (1999). A set of 400 pictures standardized for French: Norms for name agreement, image agreement, familiarity, visual complexity, image variability, and age of acquisition. $\mathrm{Be}$ havior Research Methods, Instruments, \& Computers, 31, 531-552. Attneave, F. (1954). Some informational aspects of visual perception. Psychological Review, 61, 183-193. 
Behrmann, M., \& Kimchi, R. (2003). What does visual agnosia tell us about perceptual organization and its relationship to object perception? Journal of Experimental Psychology: Human Perception \& Performance, 29, 19-42.

BERgEvin, R., \& Bubel, A. (2003). Object-level structured contour map extraction. Computer Vision \& Image Understanding, 91, 302-334.

BIEDERMAN, I. (1987). Recognition-by-components: A theory of human image understanding. Psychological Review, 94, 115-147.

Biederman, I. (1988). Aspects and extensions of a theory of human image understanding. In Z. W. Pylyshyn (Ed.), Computational processes in human vision: An interdisciplinary perspective (pp. 370428). Norwood, NJ: Ablex.

Biederman, I., \& Cooper, E. E. (1991). Priming contour-deleted images: Evidence for intermediate representations in visual object recognition. Cognitive Psychology, 23, 393-419.

BilodeaU, G.-A., \& Bergevin, R. (2002). Part segmentation of objects in real images. Pattern Recognition, 35, 2913-2926.

Braunstein, M. L., Hoffman, D. D., \& Saidpour, A. (1989). Parts of visual objects: An experimental test of the minima rule. Perception, 18, $817-826$.

Cronin, T. M. (2003). Visualizing concave and convex partitioning of 2D contours. Pattern Recognition Letters, 24, 429-443.

Cycowicz, Y. M., Friedman, D., Rothstein, M. [A.], \& Snodgrass, J. G. (1997). Picture naming by young children: Norms for name agreement, familiarity and visual complexity. Journal of Experimental Child Psychology, 65, 171-237.

Cycowicz, Y. M., Friedman, D., Snodgrass, J. G., \& Rothstein, M. [A.] (2000). A developmental trajectory in implicit memory is revealed by picture fragment completion. Memory, 8, 19-35.

Dell'Acqua, R., LotTo, L., \& Job, R. (2000). Naming times and standardized norms for the Italian PD/DPSS set of 266 pictures: Direct comparisons with American, English, French, and Spanish published databases. Behavior Research Methods, Instruments, \& Computers, 32, 588-615.

Delvenne, J.-F., Seron, X., Coyette, F., \& Rossion, B. (2004). Evidence for perceptual deficits in associative visual (prosop)agnosia: A single-case study. Neuropsychologia, 42, 597-612.

De Winter, J., Panis, S., \& Wagemans, J. (2002, May). Perceptual saliency of points along the contour of everyday objects: A largescale study. Poster presented at the meeting of the Vision Sciences Society, Sarasota, FL.

De Winter, J., Panis, S., \& Wagemans, J. (2004). Identification of everyday objects on the basis of straight-line versions. Manuscript in preparation

De Winter, J., \& Wagemans, J. (1999, August). Visual segmentation of 88 outlines of everyday objects: Bottom-up versus top-down and contours versus shapes. Paper presented at the European Conference on Visual Perception, Trieste.

De Winter, J., \& Wagemans, J. (2001, May). Segmentation of object outlines into parts: From a large-scale normative study to a tentative model. Poster presented at the meeting of the Vision Sciences Society, Sarasota, FL.

De Winter, J., \& Wagemans, J. (2003, September). Segmentation of object outlines into parts: A scale-space analysis. Poster presented at the European Conference on Visual Perception, Paris.

De Winter, J., \& Wagemans, J. (2004a). Perceptual saliency of points along the contour of everyday objects: A large-scale study. Manuscript in preparation.

De Winter, J., \& Wagemans, J. (2004b). Segmentation of object outlines into parts: From a large-scale study to an integrative framework. Manuscript submitted for publication.

DhANDAPANI, R., \& Kimia, B. B. (2002). Role of scale in partitioning shape. In Proceedings of the International Conference on Image Processing II (pp. 565-568). Piscataway, NJ: IEEE Signal Processing Society.

Elder, J. H., \& GoldBerg, R. M. (2001). Image editing in the contour domain. IEEE Transactions on Pattern Analysis \& Machine Intelligence, PAMI-23, 291-296.

Elder, J. H., KrupniK, A., \& Johnston, L. A. (2003). Contour grouping with prior models. IEEE Transactions on Pattern Analysis \& Machine Intelligence, PAMI-25, 661-674.
Feldman, J. (2001). Bayesian contour integration. Perception \& Psychophysics, 63, 1171-1182.

FreiXenet, J., Muñoz, X., Raba, D., Martí, J., \& Cufi, X. (2002). Yet another survey on image segmentation: Region and boundary information integration. Lecture Notes in Computer Science, 2352, 408422.

Giersch, A., Humphreys, G. W., Boucart, M., \& Kovacs, I. (2000). The computation of occluded contours in visual agnosia: Evidence for early computation prior to shape binding and figure-ground coding. Cognitive Neuropsychology, 17, 731-759.

HAYWARD, W. G. (1998). Effects of outline shape in object recognition. Journal of Experimental Psychology: Human Perception \& Performance, 24, 427-440.

Hayward, W. G., Tarr, M. J., \& Corderoy, A. K. (1999). Recognizing silhouettes and shaded images across depth rotation. Perception, 28, 1197-1215.

Hirshman, E., Snodgrass, J. G., Mindes, J., \& Feenan, K. (1990). Conceptual priming in fragment completion. Journal of Experimental Psychology: Learning, Memory, \& Cognition, 16, 634-647.

Hoffman, D. D., \& Richards, W. A. (1984). Parts of recognition. Cognition, 18, 65-96.

Hoffman, D. D., \& Singh, M. (1997). Salience of visual parts. Cognition, 63, 29-78.

JIANG, X. Y. (2000). An adaptive contour closure algorithm and its experimental evaluation. IEEE Transactions on Pattern Analysis \& Machine Intelligence, PAMI-22, 1252-1265.

Kellman, P. J. (2003). Interpolation processes in the visual perception of objects. Neural Networks, 16, 915-923.

Kennedy, J. M., \& Domander, R. (1985). Shape and contour: The points of maximum change are least useful for recognition. Perception, 14, 367-370.

Koenderink, J. J., \& van Doorn, A. J. (1982). The shape of smooth objects and the way contours end. Perception, 11, 129-137.

Kremin, H., Akhutina, T., Basso, A., Davidoff, J., De Wilde, M., Kitzing, P., Lorenz, A., Perrier, D., van der Sandt-Koenderman, M., Vendrell, J., Weniger, D., Apt, P., Arabia, C., De Bleser, R., Cohen, H., Corbineau, M., Dolivet, M. C., Hirsh, K., LehouX, E., Metz-Lutz, M. N., Montanes, P., Plagne, S., Polonskaya, N., Sirois, M., Stachowiak, F., Sweeney, T., \& Vish-Brink, E. (2003). A cross-linguistic data bank for oral picture naming in Dutch, English, German, French, Italian, Russian, Spanish, and Swedish (PEDOI). Brain \& Cognition, 53, 243-246.

LEEPER, R. (1935). A study of a neglected portion of the field of learning: The development of sensory organization. Journal of Genetic Psychology, 46, 41-75.

LLOYD-JoNES, T. J., \& LUCKHURST, L. (2002). Outline shape is a mediator of object recognition that is particularly important for living things. Memory \& Cognition, 30, 489-498.

Lowe, D. G. (1985). Perceptual organization and visual recognition. Boston: Kluwer.

Lowe, D. G. (1987). Three-dimensional object recognition from twodimensional images. Artificial Intelligence, 31, 355-395.

LowE, D. G. (1989). Organization of smooth image curves at multiple scales. International Journal of Computer Vision, 3, 119-130.

MarTeIN, R. (1995). Norms for name and concept agreement, familiarity, visual complexity and image agreement on a set of 216 pictures. Psychologica Belgica, 35, 205-225.

Norman, J. F., Phillips, F., \& Ross, H. E. (2001). Information concentration along the boundary contours of naturally shaped solid objects. Perception, 30, 1285-1294.

Panis, S., De Winter, J., \& Wagemans, J. (2002, May). Identification of everyday objects on the basis of contour fragments: Salient points are less useful than midpoints. Poster presented at the meeting of the Vision Sciences Society, Sarasota, FL.

Panis, S., De Winter, J., \& Wagemans, J. (2004). Identification of everyday objects on the basis of contour fragments. Manuscript in preparation.

Piccini, C., Lauro-Grotto, R., Del Viva, M. M., \& Burr, D. (2003). Agnosia for global patterns: When the cross-talk between grouping and visual selective attention fails. Cognitive Neuropsychology, 20, 3-25. Ploeger, A., Wagemans, J., Beckers, T., \& Vanroose, P. (1998, Au- 
gust). Are silhouettes easier to recognize than outlines? Poster presented at the European Conference on Visual Perception, Oxford.

Richards, W. A., \& HofFMAN, D. D. (1985). Codon constraints on closed 2D shapes. Computer Vision \& Image Processing, 31, 265-281.

Rosin, P. L. (2000). Shape partitioning by convexity. IEEE Transactions on Systems, Man, \& Cybernetics—Part A: Systems \& Humans, SMC30, 202-210.

Rossion, B., \& Pourtois, G. (2004). Revisiting Snodgrass and Vanderwart's object pictorial set: The role of surface detail in basic-level object recognition. Perception, 33, 217-236.

Sanfeliu, M. C., \& Fernandez, A. (1996). A set of 254 SnodgrassVanderwart pictures standardized for Spanish: Norms for name agreement, image agreement, familiarity, and visual complexity. Behavior Research Methods, Instruments, \& Computers, 28, 537-555.

SidDIQI, K., \& Kimia, B. B. (1995). Parts of visual form: Computational aspects. IEEE Transactions on Pattern Analysis \& Machine Intelligence, PAMI-17, 239-251.

Siddiqi, K., Tresness, K. J., \& Kimia, B. B. (1996). Parts of visual form: Psychophysical aspects. Perception, 25, 399-424.

Singh, M., Seyranian, G. D., \& Hoffman, D. D. (1999). Parsing silhouettes: The short-cut rule. Perception \& Psychophysics, 61, 636660 .

SnODgrass, J. G., \& CoRwIN, J. (1988). Perceptual identification thresholds for 150 fragmented pictures from the Snodgrass and Vanderwart picture set. Perceptual \& Motor Skills, 67, 3-36.

SnOdgrass, J. G., \& FeEnan, K. (1990). Priming effects in picture fragment completion: Support for the perceptual closure hypothesis. Journal of Experimental Psychology: General, 119, 276-296.

Snodgrass, J. G., \& Hirshman, E. (1994). Dissociations among implicit and explicit memory tasks: The role of stimulus similarity. Journal of Experimental Psychology: Learning, Memory, \& Cognition, 20, 150-160.

Snodgrass, J. G., Hirshman, E., \& FAn, J. (1996). The sensory match effect in recognition memory: Perceptual fluency or episodic trace? Memory \& Cognition, 24, 367-383.

SNODGRAss, J. G., \& VANDERWART, M. (1980). A standardized set of 260 pictures: Norms for name agreement, image agreement, familiarity, and visual complexity. Journal of Experimental Psychology: Learning, Memory, \& Cognition, 6, 174-215.

Snodgrass, J. G., \& Yuditsky, T. (1996). Naming times for the Snodgrass and Vanderwart pictures. Behavior Research Methods, Instruments, \& Computers, 28, 516-536.

Street, R. F. (1931). A gestalt completion test: A study of a cross section of intellect. New York: Columbia University, Teachers College, Bureau of Publications.

Tulving, E., \& Schacter, D. L. (1990). Priming and human memory systems. Science, 247, 301-306.

Ullman, S. (1989). Aligning pictorial descriptions: An approach to object recognition. Cognition, 32, 193-254.

Vaina, L. M., \& ZlateVA, S. D. (1990). The largest convex patches: A boundary-based method for obtaining object parts. Biological $\mathrm{Cy}$ bernetics, 62, 225-236.

Van Schagen, I., Tamsma, N., Bruggemann, F., Jackson, J. L., \& Michon, J. A. (1983). Namen en normen voor plaatjes [Names and norms for pictures]. Nederlands Tijdschrift voor de Psychologie, 38, 236-241.

Viggiano, M. P., \& Kutas, M. (2000). Overt and covert identification of fragmented objects inferred from performance and electrophysiological measures. Journal of Experimental Psychology: General, 129, 107-125.

Wagemans, J., De Winter, J., Op de Beeck, H., Ploeger, A., BeckERS, T., \& VANROOSE, P. (2004). Identification of everyday objects on the basis of silhouette and outline versions. Manuscript in preparation.

Wagemans, J., De Winter, J., \& PAnis, S. (2002, May). The awakening of Attneave's sleeping cat: Identification of everyday objects on the basis of straight-line versions. Poster presented at the meeting of the Vision Sciences Society, Sarasota, FL.

Wagemans, J., Notebaert, W., Beckers, T., \& Vanroose, P. (1997, November). Picture identification of everyday objects: Silhouettes, outlines, and contour fragments. Paper presented at the meeting of the Psychonomic Society, Philadelphia.
Wagemans, J., Notebaert, W., \& Boucart, M. (1998). Lorazepam but not diazepam impairs identification of pictures on the basis of specific contour fragments. Psychopharmacology, 138, 326-333.

Wagemans, J., Panis, S., De Winter, J., \& Op De Beeck, H. (2001, May). Perceptual and conceptual priming in picture identification on the basis of contour fragments with specific curvature properties. Poster presented at the 1st meeting of the Vision Sciences Society, Sarasota, FL.

Witkin, A. P. (1986). Scale-space filtering. In A. P. Pentland (Ed.), From pixels to predicates: Recent advances in computational and robotic vision (pp. 5-19). Norwood, NJ: Ablex.

\section{NOTE}

1. The distinction between norms and data is not always clear. We think this is a matter of how the results are used rather than of strict criteria such as the number of participants (which is always relatively large in the studies reported in this paper in comparison with related studies in the literature). In this article, when the results support a theoretical point or raise additional theoretical questions, we use the term data. When we believe our results can be used to select a particular subset of our stimuli for particular purposes (e.g., with specific levels of identifiability), we will describe them as norms.

\section{ARCHIVED MATERIALS}

The following materials and links may be accessed through the Psychonomic Society's Norms, Stimuli, and Data archive, http://www. psychonomic.org/archive/. To access these files or links, search the archive for this article using the journal (Behavior Research Methods, Instruments, \& Computers), the first author's name (De Winter), and the publication year (2004).

FILE: DeWinter-BRMIC-2004-Otl.zip.

DESCRIPTION: The compressed archive file (23M) contains six files: Silhouettes BMP.zip, containing stimuli developed in the present article, as a $796 \mathrm{~K}$ compressed archive file containing 260 Windows BMP files of silhouettes derived from 260 line drawings from Snodgrass and Vanderwart (1980).

Outlines DIF.zip, containing stimuli developed in the present article, as a $1,726 \mathrm{~K}$ compressed archive file containing 260 tab-delimited text files of outlines derived from the above silhouettes.

Outlines BMP.zip, containing stimuli developed in the present article, as a $756 \mathrm{~K}$ compressed archive file containing 260 Windows BMP files of outlines derived from the above silhouettes.

Outlines Smoothed DIF.zip, containing stimuli developed in the present article, as an $18 \mathrm{M}$ compressed archive file containing $3 * 260$ tabdelimited text files with Gaussian smoothed outlines. For each point on the outline; Columns 1 and 2 contain the $x, y$ coordinates, and Column 3 , the curvature value.

Outlines Smoothed BMP.zip, containing stimuli developed in the present article, as a $1,000 \mathrm{~K}$ compressed archive file containing $3 * 260$ Windows BMP files of Gaussian smoothed outlines.

Outlines KR1.zip, containing stimuli developed in the present article, as a $583 \mathrm{~K}$ compressed archive file containing 260 tab-delimited text files of silhouette versions of 260 line drawings from Snodgrass and Vanderwart (1980). Columns 1 and 2 contain the $x, y$ coordinates, and Column 3 , the curvature value for each point on the outline. This set was used for the segmentation study only (so use this file to interpret the data contained in Parts.zip) and contains some duplicate points (which were later removed and resulted in the Outlines DIF.zip file, but both versions have outlines identical in shape).

FILE: DeWinter-BRMIC-2004-SL.zip.

DESCRIPTION: The compressed archive file (12M) contains eight files:

SL E\&I 1ELOB DIF.zip, containing stimuli developed in the present article, as a $294 \mathrm{~K}$ compressed archive file containing 260 tab-delimited text files used to form straight-line outlines by connecting selected points. Here, only one (corner) point per positive/negative curvature segment (lob) is selected. Columns 1 and 2 contain the $x, y$ coordinates, Column 3 the curvature value, Column 4 the cumulative outlinedistance, and Column 5 the singularity type. 
SL E\&I 1ELOB BMP.zip, containing stimuli developed in the present article, as a $698 \mathrm{~K}$ compressed archive file containing 260 Windows BMP files of straight-line outline variants, formed by selecting only one corner point per positive/negative curvature segment.

SL E\&I Count DIF.zip, containing stimuli developed in the present article, as a $268 \mathrm{~K}$ compressed archive file containing 260 tab-delimited text files used to form straight-line outlines, matching the number of corner points with the number of salient points in the Salient Points DIF.zip file. Columns 1 and 2 contain the $x, y$ coordinates, Column 3 the curvature value, Column 4 the cumulative outline-distance, and Column 5 the singularity type.

SL E\&I Count BMP.zip, containing stimuli developed in the present article, as a $627 \mathrm{~K}$ compressed archive file containing 260 Windows BMP files of straight-line outlines, matching the number of corner points with the number of salient points (SPs) of the Salient Points DIF.zip file.

SL SP\&MP DIF.zip, containing stimuli developed in the present article, as a $1,353 \mathrm{~K}$ compressed archive file containing 260 tab-delimited text files of outlines with marked SPs and midpoint (MP). These points can be used to form straight-line variants. Columns 1 and 2 contain the $x, y$ coordinates, Column 3 the curvature value for each point on the outline, Column 4 the cumulative outline distance, Column 5 the point type ( $M+$ for SP with positive curvature, $m-$ for SP with negative curvature, $I$ for MP), and Column 6 the saliency value.

SL SP\&MP BMP.zip, containing stimuli developed in the present article, as a $7,896 \mathrm{~K}$ compressed archive file containing 260 Windows BMP files of straight-line outline variants using marked SPs and MP as corner points.

SL MP-Tangent DIF.zip, containing stimuli developed in the present article, as a $78 \mathrm{~K}$ compressed archive file containing 260 tab-delimited text files of outline MP. These points can be used to form straight-line variants by drawing tangent lines thru the MP. Columns 1 and 2 contain the $x, y$ coordinates, Column 3 the curvature value, Column 4 the cumulative outline distance, Column 5 the point type, and Column 6 the saliency value.

SL MP-Tangent BMP.zip, containing stimuli developed in the present article, as a 457K compressed archive file containing 260 Windows BMP files of straight-line outlines formed by drawing tangent lines through MPs.

FILE: DeWinter-BRMIC-2004-Fr.zip.

DESCRIPTION: The compressed archive file (75M) contains eight files:

Fragments DIF.zip, containing stimuli developed in the present article, as a $32 \mathrm{M}$ compressed archive file containing 260 tab-delimited text files of outlines with fragments around SPs or MPs. These files can be used to form fragment variants by drawing only the marked points $(x$ in Column 7). Columns 1 and 2 contain the $x, y$ coordinates, Column 3 the curvature, Column 4 the cumulative outline distance, Column 5 the point type, Column 6 the saliency value, and Column 7 the marked fragment points.

Fragments BMP.zip, containing stimuli developed in the present article, as a $1,423 \mathrm{~K}$ compressed archive file containing 260 Windows BMP files of fragmented outlines formed by drawing points surrounding SPs or MPs.

SL Fragments Around DIF.zip, containing stimuli developed in the present article, as a $16 \mathrm{M}$ compressed archive file containing 260 tabdelimited text files of straight-line outlines (using SPs as corner points) with fragments around SPs or MPs. These files can be used to form straight-line fragment variants by drawing only the marked points $(x$ in Column 7). Columns 1 and 2 contain the $x, y$ coordinates, Column 3 the curvature value, Column 4 the cumulative outline distance, Column 5 the point type, Column 6 the saliency value, and Column 7 the marked fragment points.

SL Fragments Around BMP.zip, containing stimuli developed in the present article, as a $937 \mathrm{~K}$ compressed archive file containing $260 \mathrm{Win}-$ dows BMP files of fragmented straight-line outlines formed by drawing fragments surrounding SPs or MPs.

SL Fragments From DIF.zip, containing stimuli developed in the present article, as a $16 \mathrm{M}$ compressed archive file containing 260 tabdelimited text files of straight-line outlines (using SPs as corner points) with fragments starting from SPs. These files can be used to form straight-line fragment variants by drawing only the marked points $(x$ in Column 7). Columns 1 and 2 contain the $x, y$ coordinates, Column 3 the curvature value, Column 4 the cumulative outline distance, Column 5 the point type, Column 6 the saliency value, and Column 7 the marked fragment points.

SL Fragments From BMP.zip, containing stimuli developed in the present article, as a $515 \mathrm{~K}$ compressed archive file containing $260 \mathrm{Win}-$ dows BMP files of fragmented straight-line outlines formed by drawing fragments starting from SPs.

SL Fragments To DIF.zip, containing stimuli developed in the present article, as an $8 \mathrm{M}$ compressed archive file containing 260 tab-delimited text files of straight-line outlines (using SPs as corner points) with fragments ending in SPs. These files can be used to form straight-line fragment variants by drawing only the marked points ( $x$ in Column 7). Columns 1 and 2 contain the $x, y$ coordinates, Column 3 the curvature value, Column 4 the cumulative outline distance, Column 5 the point type, Column 6 the saliency value, and Column 7 the marked fragment points.

SL Fragments To BMP.zip, containing stimuli developed in the present article, as a 548K compressed archive file containing 260 Windows BMP files of fragmented straight-line outlines formed by drawing fragments ending in SPs.

FILE: DeWinter-BRMIC-2004-Dat.zip.

DESCRIPTION: The compressed archive file (1.2M) contains 17 files:

Concept Identification.zip, containing norms developed in the present article, as a $2 \mathrm{~K}$ compressed archive file containing a comma separated value (CSV) file. Each row represents one of 260 outlines/silhouettes; Column 1 represents the object number as used by Snodgrass and Vanderwart (1980), Column 2 represents the concept identification rate for outlines, and Column 3 represents the concept identification rate for silhouettes.

Salient Points.zip, containing norms developed in the present article, as a $112 \mathrm{~K}$ compressed archive file containing a CSV file. Each row represents a marked point; Column 1 represents the object number as used by Snodgrass and Vanderwart (1980), Column 2 represents the subject number, and Column 3 represents the index (starting at zero) in the outline file of the point marked by the subject.

SL E\&I 1ELOB Rate.zip, containing norms developed in the present article, as a $2 \mathrm{~K}$ compressed archive file containing a CSV file. Each row represents one of 184 straight-line variant outlines; Column 1 represents the object number as used by Snodgrass and Vanderwart (1980), Column 2 is the condition ( $\mathrm{I}=$ straight-line variant starting from selected inflections, $\mathrm{E}=$ straight-line variant starting from selected extrema), and Column 3 represents concept identification for the straightline variant outlines contained in SL E\&I 1ELOB BMP.zip.

SL E\&I 1ELOB Data.zip, containing data collected in the present article, as a $49 \mathrm{~K}$ compressed archive file containing a CSV file. Each row represents one of 184 straight-line variant outlines; Column 1 represents the object number as used by Snodgrass and Vanderwart (1980), Column 2 is the subject number, Column 3 is the condition ( $\mathrm{I}=$ straightline variant starting from selected inflections, $\mathrm{E}=$ straight-line variant starting from selected extrema), Column 4 is the subject's response (identification), Column 5 is 0 if the object identification is incorrect and 1 if the object identification is correct for the straight-line variant outlines contained in SL E\&I 1ELOB BMP.zip.

SL E\&I Count Rate.zip, containing norms developed in the present article, as a $2 \mathrm{~K}$ compressed archive file containing a CSV file. Each row represents one of 184 straight-line variant outlines; Column 1 represents the object number as used by Snodgrass and Vanderwart (1980), Column 2 is the condition ( $\mathrm{I}=$ straight-line variant starting from selected inflections, $\mathrm{E}=$ straight-line variant starting from selected extrema), and Column 3 represents concept identification for the straightline variant outlines contained in SL E\&I Count BMP.zip.

SL E\&I Count Data.zip, containing data collected in the present article, as a $43 \mathrm{~K}$ compressed archive file containing a CSV file. Each row represents one of 184 straight-line variant outlines; Column 1 represents the object number as used by Snodgrass and Vanderwart (1980), Column 2 is the subject number, Column 3 is the condition ( $\mathrm{I}=$ straightline variant starting from selected inflections, $\mathrm{E}=$ straight-line variant 
starting from selected extrema), Column 4 is the subject's response (identification), and Column 5 is 0 if the object identification is incorrect and 1 if the object identification is correct for the straight-line variant outlines contained in SL E\&I Count BMP.zip.

SL SP\&MP Rate.zip, containing norms developed in the present article, as a $4 \mathrm{~K}$ compressed archive file containing a CSV file. Each row represents one of 184 straight-line variant outlines; Column 1 represents the object number as used by Snodgrass and Vanderwart (1980), Column 2 represents the used corner point type (SP or MP), Column 3 represents the selected number of SPs (100\% or $75 \%)$, and Column 4 represents the identification rate for outlines contained in SL SP\&MP BMP.zip.

SL SP\&MP Data.zip, containing data collected in the present article, as a $95 \mathrm{~K}$ compressed archive file containing a CSV file. Each row represents one of 184 straight-line variant outlines; Column 1 represents the object number as used by Snodgrass and Vanderwart (1980), Column 2 is the subject number, Column 3 is the condition ( $\mathrm{SP}=$ straightline variant starting from SPs, MP = straight-line variant starting from MPs), Column 5 is the percentage of most salient selected SP, Column 5 is the subject's response (identification), and Column 6 is 0 if the object identification is incorrect and 1 if the object identification is correct for the straight-line variant outlines contained in SL SP\&MP BMP.zip.

SL MP Tangent Rate.zip, containing norms developed in the present article, as a $1 \mathrm{~K}$ compressed archive file containing a CSV file. Each row represents one of 142 straight-line variant outlines; Column 1 represents the object number as used by Snodgrass and Vanderwart (1980), and Column 2 represents the identification rate for outlines contained in SL MP-Tangent BMP.zip.

SL MP Tangent Data.zip, containing data collected in the present article, as a $14 \mathrm{~K}$ compressed archive file containing a CSV file. Each row represents one of 142 straight-line variant outlines; Column 1 represents the object number as used by Snodgrass and Vanderwart (1980), Column 2 is the subject number, Column 3 is the subject's response (identification), and Column 4 is 0 if the object identification is incorrect and 1 if the object identification is correct for the straight-line variant outlines contained in SL MP-Tangent BMP.zip.

Fragments Rate.zip, containing norms developed in the present article, as a $6 \mathrm{~K}$ compressed archive file containing a CSV file. Each row represents one of 188 fragment variant outlines; Column 1 represents the object number as used by Snodgrass and Vanderwart (1980), Column 2 represents the used point type (around SP or MP), Column 3 represents the percentage of the outline visible as fragments $(15 \%, 20 \%$, $25 \%$, or $30 \%$ ), and Column 4 represents the identification rate for fragmented outlines contained in Fragments BMP.zip.

Fragments Data.zip, containing data collected in the present article, as a $171 \mathrm{~K}$ compressed archive file containing a CSV file. Each row represents one of 188 fragment variant outlines; Column 1 represents the object number as used by Snodgrass and Vanderwart (1980), Column 2 is the subject number, Column 3 is the condition ( $\mathrm{SP}=$ around salient points, MP = around midpoints), Column 4 represents the percentage of the outline visible as fragments $(15 \%, 20 \%, 25 \%$, or $30 \%)$, Column 5 is the subject's response (identification), and Column 6 is 0 if the object identification is incorrect and 1 if the object identification is correct for the straight-line variant outlines contained in Fragments BMP.zip.

SL Fragments Rate.zip, containing norms developed in the present article, as a $5 \mathrm{~K}$ compressed archive file containing a CSV file. Each row represents one of 188 fragmented straight-line variant outlines; Column 1 represents the object number as used by Snodgrass and Vanderwart (1980), Column 2 represents the used point type (around SP or MP), Column 3 represents the outline percentage visible as fragments $(15 \%$, $20 \%$, or $25 \%$ ), and Column 4 represents the identification rate for fragmented straight-line outlines contained in SL Fragments Around BMP.zip.

SL Fragments Data.zip, containing data collected in the present article, as a $99 \mathrm{~K}$ compressed archive file containing a CSV file. Each row represents one of 188 fragment straight-line variant outlines; Column 1 represents the object number as used by Snodgrass and Vanderwart (1980), Column 2 is the subject number, Column 3 is the condition ( $\mathrm{SP}=$ around salient points, MP = around MPs), Column 4 represents the percentage of the outline visible as fragments $(15 \%, 20 \%$, or $25 \%)$, Column 5 is the subject's response (identification), and Column 6 is 0 if the object identification is incorrect and 1 if the object identification is correct for the straight-line variant outlines contained in SL Fragments Around BMP.zip.

SL Fragments FT SP Rate.zip, containing norms developed in the present article, as a $5 \mathrm{~K}$ compressed archive file containing a CSV file. Each row represents one of 188 fragmented straight-line variant outlines; Column 1 represents the object number as used by Snodgrass and Vanderwart (1980), Column 2 represents the type of fragment (starting or ending in SP), Column 3 represents the percentage outline visible $(15 \%, 20 \%$, or $25 \%)$, and Column 4 represents the identification rate for fragmented outlines contained in SL Fragments From BMP.zip and SL Fragments To BMP.zip.

SL Fragments FT SP Data.zip, containing data collected in the present article, as a $58 \mathrm{~K}$ compressed archive file containing a CSV file. Each row represents one of 188 fragment straight-line variant outlines; Column 1 represents the object number as used by Snodgrass and Vanderwart (1980), Column 2 is the subject number, Column 3 is the condition (starting or ending in SP), Column 4 represents the percentage of the outline visible as fragments $(15 \%, 20 \%$, or $25 \%)$, Column 5 is the subject's response (identification), and Column 6 is 0 if the object identification is incorrect and 1 if the object identification is correct for the straight-line variant outlines contained in SL Fragments From BMP.zip and SL Fragments To BMP.zip.

Parts Data.zip, containing norms developed in the present article, as a $112 \mathrm{~K}$ compressed archive file containing a CSV file. Each row represents one of the segmentation lines; Column 1 represents the independent variable (easy-to-identify outlines with $>70 \%$ average identification or difficult-to-identify outlines with $<30 \%$ average identification), Column 2 represents the object number as used by Snodgrass and Vanderwart (1980), Column 3 represents the subject number, Column 4 represents the outline index point of the first segmentation point, Columns 5 and 6 represent the location of the first segmentation point $x, y, \mathrm{Col}-$ umn 7 represents the outline index point of the second segmentation point, and Columns 8 and 9 represent the location of the second segmentation point $x, y$. Use the Outlines KR1.zip outlines to interpret the data correctly.

AUTHOR'S E-MAIL ADDRESS: joeri.dewinter@psy.kuleuven.ac.be.

Author's WeB site: http://www.psy.kuleuven.ac.be/ winterj/.

(Manuscript received January 6, 2004; revision accepted for publication July 20, 2004.) 